\title{
A Strategy Based on Protein-Protein Interface Motifs May Help in Identifying Drug Off-Targets
}

\author{
H. Billur Engin, ${ }^{\dagger}$ Ozlem Keskin, ${ }^{\dagger}$ Ruth Nussinov, ${ }^{\ddagger} \S$ and Attila Gursoy ${ }^{\dagger} *$ \\ ${ }^{\dagger}$ Center for Computational Biology and Bioinformatics and College of Engineering, Koc University, Rumelifeneri Yolu, 34450 Sariyer, \\ Istanbul, Turkey \\ ${ }^{\ddagger}$ Center for Cancer Research Nanobiology Program, Frederick National Laboratory for Cancer Research, SAIC-Frederick, Maryland \\ 21702, United States \\ ${ }^{\S}$ Sackler Inst. of Molecular Medicine, Sackler School of Medicine, Tel Aviv University, Tel Aviv 69978, Israel
}

Supporting Information

ABSTRACT: Networks are increasingly used to study the impact of drugs at the systems level. From the algorithmic standpoint, a drug can "attack" nodes or edges of a proteinprotein interaction network. In this work, we propose a new network strategy, "The Interface Attack", based on proteinprotein interfaces. Similar interface architectures can occur between unrelated proteins. Consequently, in principle, a drug that binds to one has a certain probability of binding to others. The interface attack strategy simultaneously removes from the network all interactions that consist of similar interface motifs. This strategy is inspired by network pharmacology and allows inferring potential off-targets. We introduce a network model

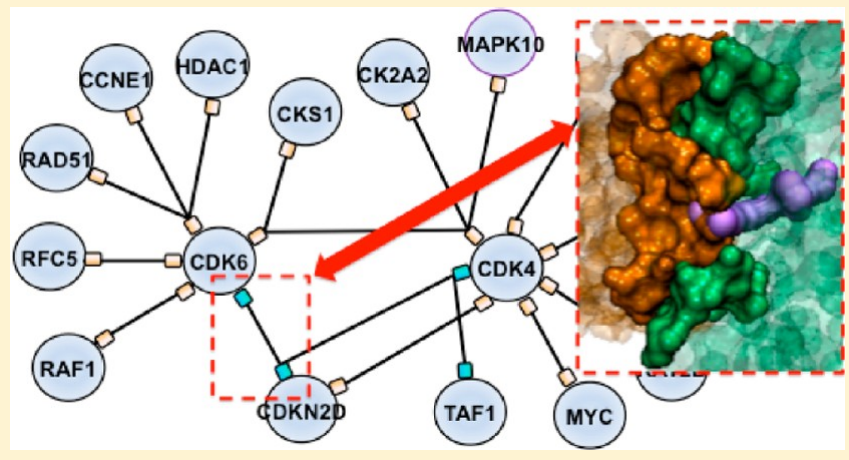
that we call "Protein Interface and Interaction Network (P2IN)", which is the integration of protein-protein interface structures and protein interaction networks. This interface-based network organization clarifies which protein pairs have structurally similar interfaces and which proteins may compete to bind the same surface region. We built the P2IN with the p53 signaling network and performed network robustness analysis. We show that (1) "hitting" frequent interfaces (a set of edges distributed around the network) might be as destructive as eleminating high degree proteins (hub nodes), (2) frequent interfaces are not always topologically critical elements in the network, and (3) interface attack may reveal functional changes in the system better than the attack of single proteins. In the off-target detection case study, we found that drugs blocking the interface between CDK6 and CDKN2D may also affect the interaction between CDK4 and CDKN2D.

\section{INTRODUCTION}

Currently, the "one drug one target" approach is often considered an exception rather than the rule. ${ }^{1}$ With a few exceptions, there is no one-to-one mapping between a gene (and the protein it encodes) and a disease state, and disease processes often involve crosstalk between proteins in different pathways. This is mainly because a protein can have many functions, and many proteins can have a similar function. Drugs are often multi-targeted, and a recent study suggested that the average number of target proteins per drug is $6.3 .^{2}$ Off-targets are the proteins that a drug can bind to other than its primary target. Cohen et al. ${ }^{3}$ note that cancer drug therapy might change the behavior of nearly 1000 different proteins, suggesting that the disturbance of a signaling network through a single protein may affect other proteins. Recently, systemwide approaches are increasingly being considered. ${ }^{4}$

Polypharmacology is a new concept in drug discovery that seeks drugs acting on multiple targets. One of the aims of polypharmacology is to discover multi-target drugs that will disturb disease-associated networks, ${ }^{1}$ similar to aspirin,
Metformin, nonsteroidal anti-inflammatory drugs (NSAIDs), and Gleevec. Combination therapy showed success in diseases such as AIDS, atherosclerosis, cancer, and depression. Although attacking more than one "strategic" point of the system might be a useful approach, multi-target drugs also have increased toxicity. Putative off-targets have been found through different computational methods, such as docking, ${ }^{5,6}$ pharmacophore mapping, ${ }^{7}$ ligand structure similarity, ${ }^{8,9}$ side effect similarity, ${ }^{10,11}$ ligand binding site similarity, ${ }^{12-17}$ text mining, ${ }^{18}$ and integrated methods. ${ }^{19-21}$ Proteins with similar binding sites often recognize similar ligands. ${ }^{21,22}$ Earlier works suggested that even weak binding to multiple targets may have profound effects on the biological system. ${ }^{23-25}$ Eventually, all structurebased drug discovery strategies including those targeting protein-protein interactions (PPIs) ${ }^{26}$ should consider protein flexibility on the atomistic level. ${ }^{27,28}$

Received: February 6, 2012

Published: July 20, 2012 

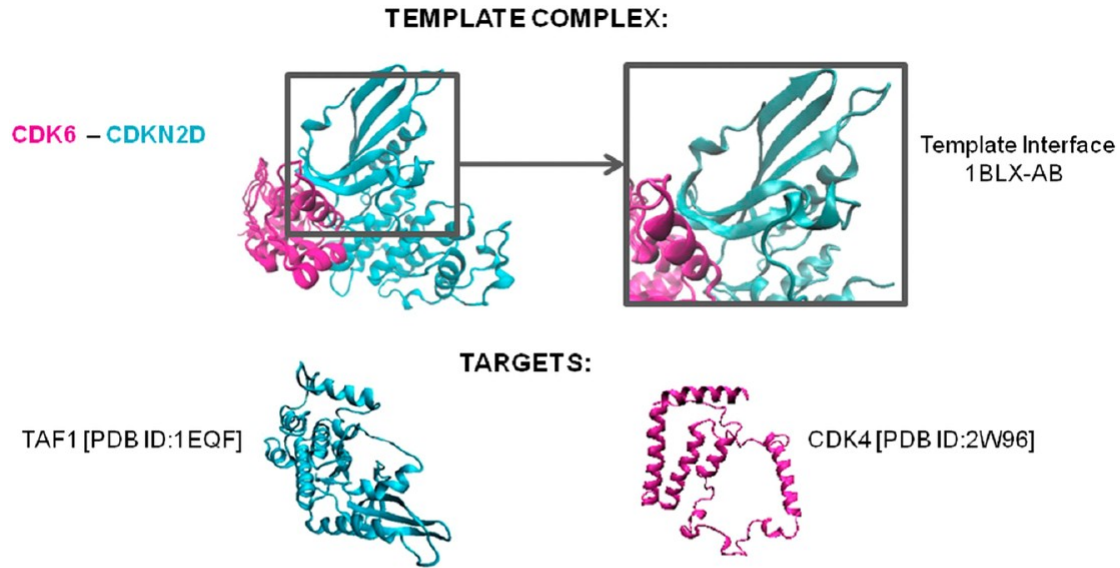

PREDICTED COMPLEX :

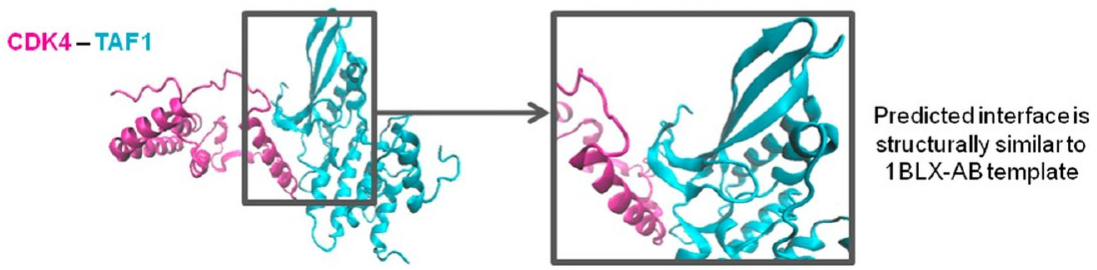

Figure 1. Interface Structure Prediction for Interacting Target Proteins. Interface information is obtained from the "Protein Interactions by Structural Matching" (PRISM) server. PRISM searches for spatial motif similarity on target proteins' surfaces using geometric complementarity and considers evolutionary conservation of hot spots on the basis of a nonredundant protein-protein interface template data set derived from the PDB. Its prediction principle is to compare both sides of a template interface with surface regions of any given two monomers, and if they are similar, these two proteins are predicted to interact with each other via this interface region. In the above example, the CDK6 [PDB:1BLX-A] and CDKN2D [PDB:1BLX-B] complex is derived from PDB, and the target proteins CDK4 and TAF1 are found to be interacting via an interface structurally similar to the $1 \mathrm{BLX}-\mathrm{AB}$ interface. CDK 4 and TAF1 are predicted to be interacting via the 1BLX-AB interface.

The interface attack strategy proposed in this work focuses on protein-protein interface motifs. Currently, proteinprotein interfaces are increasingly becoming targets in drug discovery, ${ }^{29,30}$ and it was suggested that the high flexibility of monomers may lead to overlooking small highly populated pockets that may occur when the complex forms. ${ }^{30}$ Finding small-molecule drugs that hit protein-protein interactions is still highly challenging. ${ }^{31-35}$ Although generally interfaces of PPIs $\left(\sim 1500-3000 \AA^{2}\right)$ are larger than protein-small molecule interactions $\left(\sim 300-1000 \AA^{2}\right)$, an optimized small molecule may bind with an affinity comparable to that of the native partner protein or peptide. ${ }^{32}$

Our interface attack is inspired by interface motifs and by multi-target drugs. Because drugs may disrupt protein interactions that have structurally similar interfaces, we aim to develop a strategy that may take a first step toward prediction of the outcome of disabling a set of structurally similar interactions in protein-protein interaction networks (PINs). Our study is the first to target interfaces in a network attack. A few successful PPI drugs on the market, ${ }^{34}$ such as tirofiban targeting the integrins (cardiovascular conditions) ${ }^{36}$ and maraviroc targeting CCR5-gp120 interactions (HIV) ${ }^{37}$ and several new drugs entering phase II clinical trials, ${ }^{38}$ suggest that protein interfaces can be druggable.

Biological systems are robust and are resilient to damage of their components. In the case of the protein-protein interaction network, the topology of the network appears responsible for its robustness. ${ }^{39}$ The scale-free network characteristics make it resilient to accidental failures; even if $80 \%$ of randomly selected proteins fail, the remaining $20 \%$ still continue to stay as a compact cluster. ${ }^{40}$ However, protein networks are vulnerable to systematic attacks on highly connected proteins. Experimental studies indicate that these proteins are also essential. ${ }^{41}$ Integrating structural knowledge can help the abstract protein interaction networks; knowledge of protein interfaces helps not only in understanding the relations among proteins and thus function but also in identifying drug targets in inhibitor design and off-targets prediction.

Beyond the introduction of the strategy of interface attack, this article presents a new network model, which we name "Protein Interface and Interaction Network (P2IN)". Similar network models were used by our group previously to analyze interface properties of cancer-related proteins ${ }^{42}$ and topological properties of hubs. ${ }^{43}$ This new model introduces structural information into PINs. All network nodes have 3D interface structures. This representation illustrates which proteins may compete for the same binding site on a protein and all protein pairs with structurally similar interface topologies.

Here our goal is not to design drugs; instead, we aim to introduce an interface motif-based first step in a systems strategy that seeks to identify potential side effects. Eventually, to more reliably predict drug binding, comprehensive long time scale simulations are essential. Introducing atomistic flexibility into systems-level studies is a challenging aim, beyond current computational feasibility. An alternative could be the selection of a few likely targets obtained by a strategy such as the one proposed here and subjecting them to detailed analysis.

This paper is organized as follows. The Concepts section provides an overview of the notion of similar structural motifs in interfaces, the new network model (P2IN), and the interface attack strategy. The Results and Discussion section consists of 
four case studies. The first is the P2IN model applied to the p53 signaling pathway, the second relates to off-target prediction, and the third case study focuses on comparison of node and interface attacks from a biological standpoint. These case studies are followed by the robustness measures used in analyzing the response of the p53 P2IN to attack strategies. Lastly, the case study on comparison of network attack strategies is presented.

\section{CONCEPTS}

The novelty of our strategy comes from the concept of similar interfaces. Similar interfaces can occur between unrelated proteins, and this is a common occurrence. ${ }^{44,45}$ Consequently, a drug that binds to one has a certain probability of binding to others. Our network attack strategy is inspired by multi-target drugs and makes use of structurally similar interfaces. In this section, we give a brief overview of this similar-interfacesdifferent-global-structures paradigm. We describe how we find similar interfaces on a network of protein interactions, introduce the structural protein-protein interaction network model, explain what a network attack is, and define interface attack, which is the focus of this study.

PRISM May Discover Similar Interfaces. The 3D structures of the protein-protein complexes and their interfaces are obtained through the application of the Protein Interactions by Structural Matching (PRISM) method. PRISM aims to predict protein-protein interactions and protein interfaces by identifying structural similarities between protein surfaces and known interfaces that are derived from the Protein Databank (PDB). ${ }^{46}$ The algorithm has four steps. First, the surfaces of all target proteins are extracted. Second, using the MultiProt engine, ${ }^{47}$ the surfaces of the target proteins are structurally aligned with known interfaces (templates) obtained from the PDB. In this step, PRISM checks whether any surface region of the monomers is structurally similar to one of the complementary chains of the template interfaces, disregarding the order of the residues in the protein chain. Third, it places the two chains that are structurally similar to the template interface onto the template complex. This leads to a putative complex. The fourth step involves flexible refinement of the putative complexes by FiberDock. ${ }^{48,49}$ This resolves steric clashes and ranks the predicted protein complexes by their energies. Combining geometric complementarity with docking tools makes the prediction more physical. The prediction performance of the PRISM algorithm was recently analyzed on standard docking benchmarks and was found to be comparable to other rigid docking strategies, however, considerably more efficient (see Tuncbag et al. ${ }^{58}$ ).

PRISM finds the similarity scores between the surface of each target in our data sets and each side of a PDB template (a template has two sides, i.e., the two complementary surfaces in the complex, in cyan and magenta, Figure 1, top line). From this output, it predicts the interface (Figure 1, bottom line). For instance, take the target protein pair in Figure 1, "TAF1" and "CDK4", and template interface "1BLX-AB"; if "TAF1" has a region on its surface that is similar to the binding site on one chain of "1BLX-AB" and "CDK4" on the second chain, then they are predicted to interact similar to the interface "1BLX$A B$ ". This means that the binding sites of proteins "TAF1" and "CDK4" are similar to those of the protein chains of interface "1BLX-AB".

A New Structural PPI Network Model: Protein Interface and Interaction Network (P2IN). Protein interaction networks (PINs) give binary information relating to whether two proteins communicate. Being enriched with structural information, P2IN is a more physical and realistic version of PIN. Unlike the PINs whose nodes are proteins and the interactions are the connecting edges, P2IN has interface information linked to its edges and each protein in the network has a 3D structure. Interactions between the proteins are represented by edges going through the interfaces of the two chains (Figure 2). Similar interfaces may exist between different protein pairs, and the same protein pair may interact through different interfaces. ${ }^{44,51,52}$

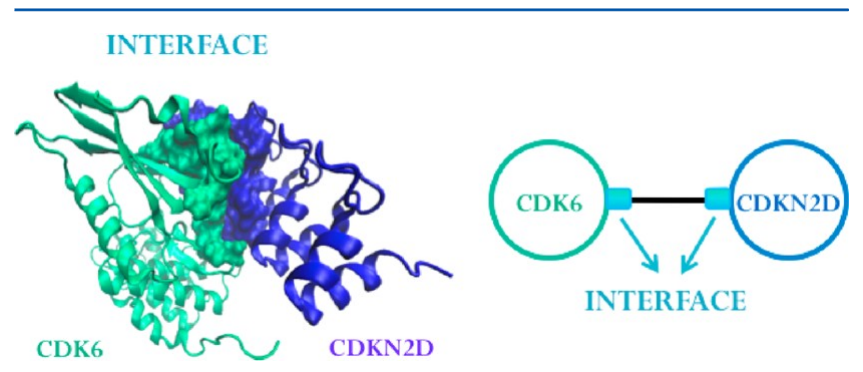

Figure 2. The P2IN Representation. Interactions between proteins are represented by the edges going through the interfaces whose two chains represent the binding site regions of the proteins.

P2IN is capable of providing structural details that a PIN is not able to describe. Some of these details are exemplified in Figure 3: different protein pairs interacting via the same interface (CDK6-CDKN2D and CDK4-TAF1 interact via same interface), a protein pair interacting using different interfaces (CDKN2D and CDK4), and multiple proteins competing to bind the same region on a protein (RAD51, CCNE1, and HDAC1 going for the same binding site on CDK6). This additional knowledge may allow identification of interactions that cannot take place simultaneously. Partners of a protein interacting with the same binding site cannot coexist. In addition, because ligands tend to bind proteins that have similar binding sites, ${ }^{53-55}$ locating protein pairs that interact via similar interfaces may help to predict additional, off-targets of these drugs. Thus, P2IN might be one step closer to mimicking systems-wise drugs effects. ${ }^{56}$

Network Attacks. An attack on a network is executed in order to disrupt the information flow locally or globally, to disable a pathway, or to destroy the network as a whole. An attack implies deletion or attenuation of an edge or a node of the network. ${ }^{25}$

i. Node Attack. A node attack on the network removes edges focused at a single node. We studied two different node attacks: complete knockout (Figure 4a) and partial knockout (Figure $4 b$ ). Complete knockout refers to removing a node with all of its edges; partial attack involves removing randomly selected half of the edges of a node. Complete node attacks are commonly used attack strategies. The targets of these attacks vary according to the network topology. Complex networks were believed to be randomly linked ${ }^{57}$ until Barabasi et al. discovered a common topology. ${ }^{58}$ This discovery introduced scale-free networks into network theory. While random network nodes have roughly the same number of edges, in a scale-free network, there are many nodes with a small number of edges and a few nodes (hubs) with a large number of connections. Random node attacks may be destructive to networks that are randomly linked, whereas scale-free networks 


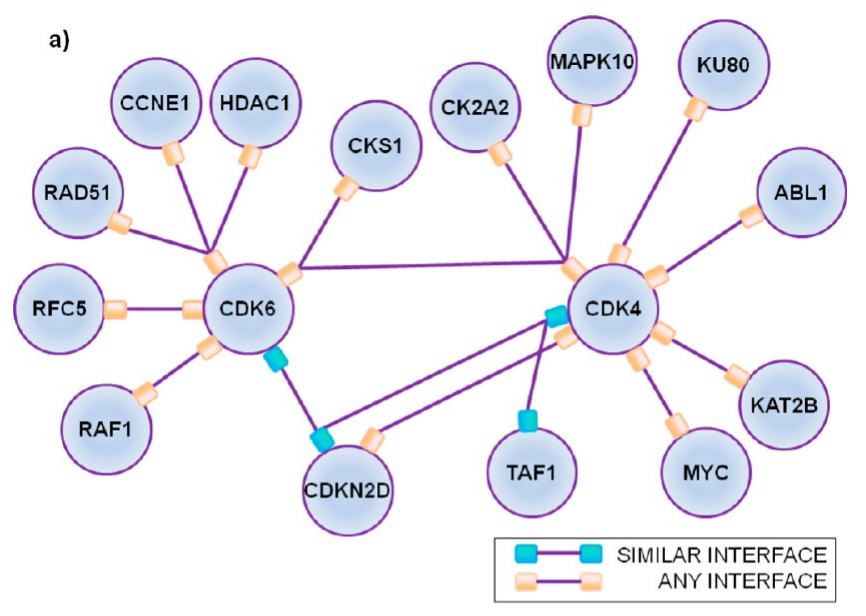

b)

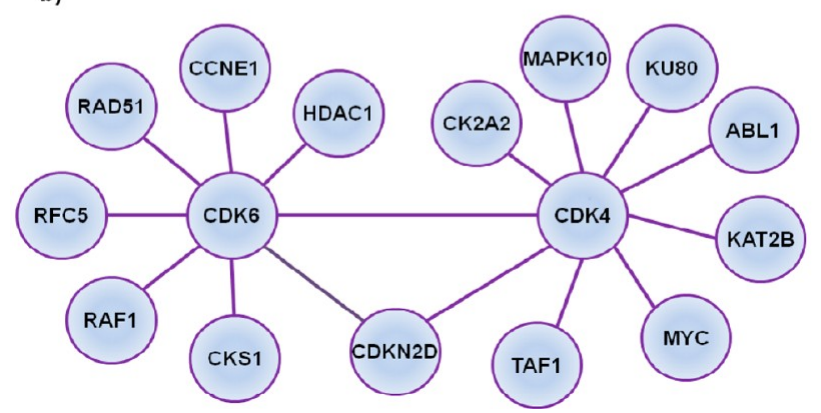

Figure 3. Protein-Protein Interactions and Interface Networks (P2IN) vs Protein-Protein Interaction Network (PIN). (a) A subset of PRISM predictions represented with P2IN and (b) its PIN counterpart. In P2IN, the same interface may exist between different protein pairs (CDK6-CDKN2D; CDK4-TAF1 interact via same interface), and the same protein pair may interact using different interfaces (CDKN2D and CDK4). Moreover, many proteins may compete to bind the same binding on a protein (RAD51, CCNE1, and HDAC1 bind the same site on CDK6). PINs are not capable of depicting such structural information of protein interactions.

are highly robust under these attacks. Scale-free networks are defenseless upon a few vital node removals. ${ }^{39}$ Accordingly, targeting hub nodes is a preferred approach in network attacks. $^{39-62}$ Detaching those nodes, which have many neighbors, will disrupt the information flow.

Partial knockout was performed by Agoston et al. ${ }^{25}$ on E. coli and $S$. cerevisiae networks. They removed randomly half of the edges of the target node or attenuated all the edges of the node. This study suggested that partial weakening of a small number of nodes (3-5) might have a stronger effect than completely removing a selected node; in both cases, the most damaging nodes were selected. Zhang et al. ${ }^{63}$ questioned whether this result is a general concept for complex networks and retested all attack strategies on the Barabasi-Albert (BA) scale-free network ${ }^{58}$ and the Erdös-Renyi (ER) random network. ${ }^{57}$ They confirmed that multi-target partial attacks may disturb complex networks more than single target complete attacks and that ER random networks are more resistant to multi-target partial attacks than the BA networks.

ii. Edge Attack. An edge attack removes one or multiple edges from the network, where the edges do not have to be incident to a node. Depending on the network topology, attacking a high betweenness edge may damage the system more than attacking a hub node with many edges. Thus,
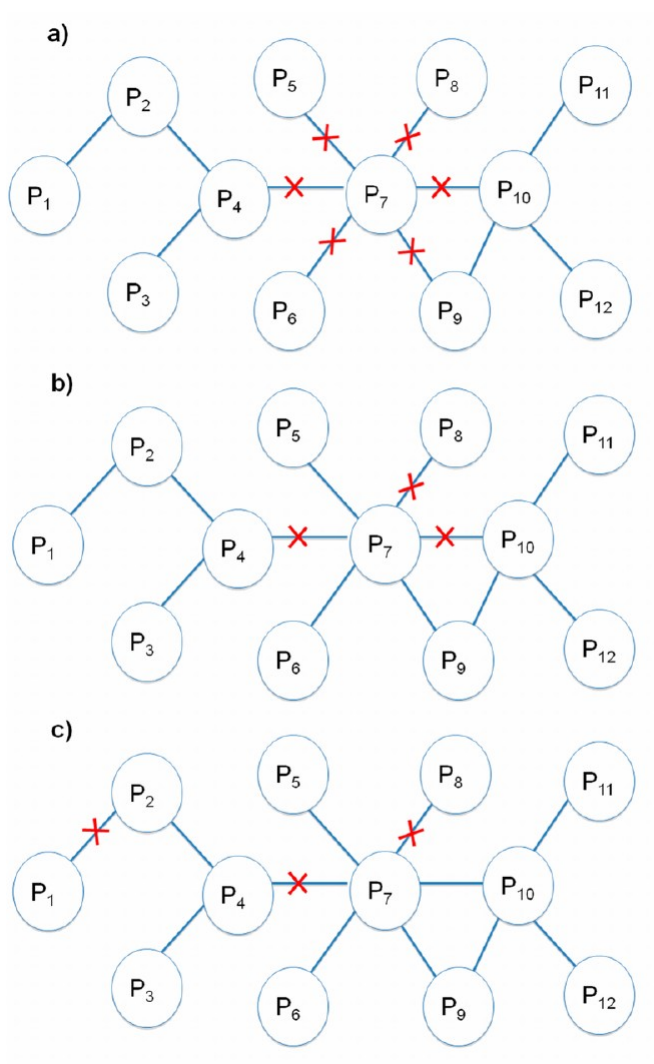

Figure 4. Network Attacks. (a) Complete knockout and (b) partial knockout target a hub node. (c) Distributed attack.

deleting a number of edges scattered in different regions of the network might be a more efficient attack strategy than targeting a node. ${ }^{25}$ This attack is a "distributed attack" (Figure 4c).

Interface Attack. Here we propose an attack strategy that is based on the expectation that PPI-targeting drugs may disrupt a number of protein-protein interactions that have structurally similar interfaces. Interface attack is the graphical representation of this strategy and removes interactions with similar interfaces from the network (Figure 5).

Interface attack is a kind of distributed attack because it targets one or more interactions between protein pairs. However, instead of selecting random edges or the ones that lead to the most damage, structurally similar interfaces are

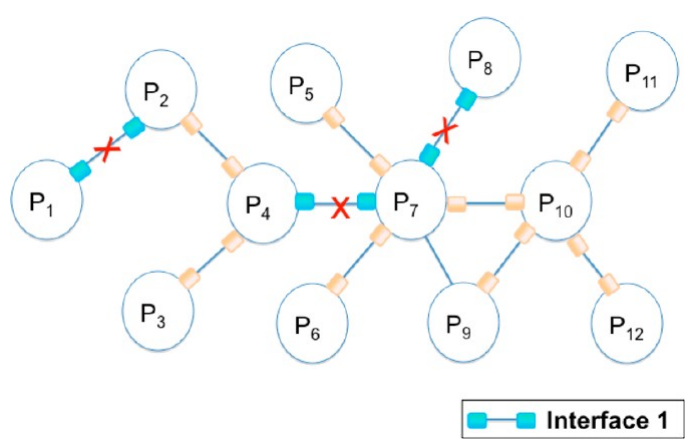

Figure 5. Interface Attack. Interface attack hits the set of edges, which interact via structurally similar interfaces (marked with red crosses). When the interaction between P1 and P2 is targeted, the interactions between $\mathrm{P} 4$ and $\mathrm{P} 7$ and $\mathrm{P} 7$ and $\mathrm{P} 8$ are also hit because they all interact through interface 1 . 
targeted. Interface attack is a knowledge-based distributed attack.

\section{RESULTS AND DISCUSSION}

As a first case study, we built the p53 protein interface and interaction network (P2IN) showing how knowledge of similar interfaces may help to detect drug off-targets. A second case study compares the consequences of the interface attack and the complete node attack. To obtain the hypothetical biological impact of interface attack, we built a new network in which the interactions and nodes without structural knowledge were also present. Lastly, the network attack scenarios are practiced on P53 P2IN and changes in the network robustness are analyzed.

P53 P2IN. The p53 tumor suppressor is a center of a protein interaction network. Under cellular stress, it is a key factor in the decision between cell cycle progression or apoptosis. ${ }^{61}$ Stress signals may be due to failures in DNA replication, chromosome segregation, and cell division. ${ }^{64}$ Malfunction of p53 causes uncontrolled growth. ${ }^{65}$ p53 is inactivated in more than $50 \%$ of human cancers. ${ }^{66,67}$ We constructed the p53 signaling P2IN using the PRISM ${ }^{68,69}$ predictions for this signaling pathway. Our network has 251 interactions among 81 proteins (refer to Table SI1 of the Supporting Information for the list of PRISM interaction predictions for p53 network). Forty-six different types of interface structures are observed for these interactions. Twenty-six out of the 251 are present in Kohn's molecular interaction map (MIM); ${ }^{70} 59$ are in PPI databases such as HPRD, ${ }^{71}$ Mint, ${ }^{72}$ IntAct, ${ }^{73}$ Reactome, ${ }^{74}$ BioGrid, ${ }^{75}$ Pathway Commons, ${ }^{76}$ and NCI-Nature PID. ${ }^{77}$ Sixtysix interaction predictions are directly experimentally validated, and there is evidence in the STRING ${ }^{78}$ database for 90 of the interactions predicted by PRISM. Overall, 104 interactions out of 251 are validated experimentally or via STRING.

Knowledge of Similar Interfaces May Help Off-Target Detection. This section describes a case study for off-target prediction application on the interfaces of p53 P2IN. CDK6 is a regulator of cell cycle progression and affects the activity of tumor suppressor protein $\mathrm{RB}$ that inhibits it and keeps the cell growing in G1 phase. Inactivation through phosphorylation by CDK leads to cell cycle progression. Some CDK6 inhibitors that block the G1/S transition of cell are listed in Table 1. The drugs in this table have 3D structures in complex with CDK6. ${ }^{46}$

Table 1. List of CDK6 Inhibitors

\begin{tabular}{|c|c|c|c|}
\hline Inhibitor Name & Resource & PUBCHEM ID ${ }^{79}$ & PDB ID \\
\hline Aminopurvalanol $^{80}$ & PDB & 6914609 & $2 \mathrm{~F} 2 \mathrm{C}$ \\
\hline PD-0332991 ${ }^{81}$ & $\mathrm{TTD},{ }^{82} \mathrm{PDB}$ & 5330286 & 2EUF \\
\hline CHEBI: $792519^{83}$ & $\mathrm{PDB}$ & 49800099 & $3 N U P$ \\
\hline CHEBI: $792520^{83}$ & PDB & 49800100 & $3 N U X$ \\
\hline Fisetin $^{84}$ & Uniprot $^{85}$ & 5281614 & $1 \mathrm{XO} 2$ \\
\hline
\end{tabular}

CDKN2D is a cyclin dependent kinase inhibitor, which forms a stable complex with CDK6 (Figure 6a). The drugs listed in the table (Aminopurvalanol, PD-0332991, CHEBI: 792519, CHEBI: 792520, and Fisetin) seem to interfere with the CDK6 and CDKN2D interface when the CDK6CDKN2D complex is superimposed on CDK6 and drug complexes present in PDB (Figure 6b,c, Figure SI1, Supporting Information). The crystal structure of the CDK6 and CDKN2D interface is available (PDB ID: 1BLX, chains: A, B. ${ }^{86} 1 \mathrm{BLX}$ is a complex between human CDK6 and mouse CDKN2D. The same complex is also available for human
CDK6 and human CDKN2D (PDB ID: 1BI8, chains: A, B) ${ }^{87}$ We considered the mouse and human CDKN2D as homologues, with $87 \%$ sequence similarity and $0.41 \mathrm{rmsd}$ and used the 1BLX complex in this study because it has a better Xray resolution. PRISM predicts an interaction between CDK4 and CDKN2D, with a structurally similar interface to the CDK6-CDKN2D interface. The interaction of CDK4 and CDKN2D is detected by in vitro and in vivo assays, ${ }^{88}$ but the $3 \mathrm{D}$ structure of their complex is unavailable. The interface attack by the five drugs blocking the interaction of CDK6CDKN2D may disturb the CDK4-CDKN2D interaction.

Using the HotPoint server, ${ }^{89}$ we identified the computational hotspots of CDK4, CDK6, and CDKN2D. When the interfaces with CDKN2D are superimposed by using the Multiprot engine, ${ }^{47}$ CDK4 (obtained from PRISM predictions) and CDK6 (obtained from the PDB) have a number of identical hotspots (Figure 7). CDKN2D interacts with them via the same surface area. Lastly, we found that the hotspot (CDK6 residue Ile19) that is closest to the ligand binding region on CDK6 is also present on the binding region of CDK4 (residue Ile12) (Figures 8 and 9). These drugs are also close to hotspots Gln98 and Asp97 on CDK4 and Gln103 (hotspot) and Asp102 (nonhotspot) on CDK6 (refer to Figures SI2 and SI3 of the Supporting Information). These residues overlap when CDK4 (PDB ID: 2W96, chain: B) and CDK6 (PDB ID: 1BLX, chain: A) are superimposed with the Multiprot engine (rmsd: $1.28 \AA$ ). At this point, we propose that CDK4 may be an off-target candidate for drug targeting CDK6. In order to see how alike the binding pockets of CDK4 and CDK6 are, we superimposed the ligand binding sites using $\mathrm{VMD}^{90}$ (Figure SI4, Supporting Information). The results revealed that CDK4 has a binding pocket that is similar to that of CDK6 with rmsd $0.87 \AA$.

Docking simulations may suggest if a ligand is capable of binding to a protein. AutoDock ${ }^{91}$ is used to dock these drugs to candidate off-target CDK4 (Figure 8) and primary target CDK6 (Figure 9). As shown in Table 2, the binding free energies between CDK4 and the drugs are promising; they are comparable to the binding energies between CDK6 and its inhibitors. The listed energies are the lowest binding free energies of the most populated clusters. The rmsd values of superimpositions of the best poses of each drug molecule docked to CDK4 compared to CDK6 are also provided in Table 2 (Figure SI5, Supporting Information). These findings strengthen our proposition that CDK4 is an off-target for the drugs targeting CDK6.

Lastly, we searched for the inter-relationship between CDK6 inhibitors and CDK4 in the literature. We found that PD0332991 has been designed to turn off both CDK4 and CDK6. ${ }^{92}$ Moreover, SuperTarget states that CDK4 is a target of CHEBI: $792520 .^{93}$ Accordingly, we are able to verify two of our off-target predictions. To conclude, we may now suggest that CDK6 binding drugs that block the interface between CDK6 and $\mathrm{CDKN} 2 \mathrm{D}$ may also bind to CDK4 and disrupt the interaction between CDK4 and CDKN2D. Therefore, when the CDK6-CDKN2D interaction is hit in the interface attack, we may also break the interaction between CDK4 and CDKN2D.

Biological Consequences of Interface Attack vs Complete Node Attack. Networks of protein interactions are vital tools for explaining a series of events in the cell that may be triggered by a drug. A drug that inhibits proteinprotein interactions may be represented in the network by removing the respective edges. To foresee the effects of a drug 

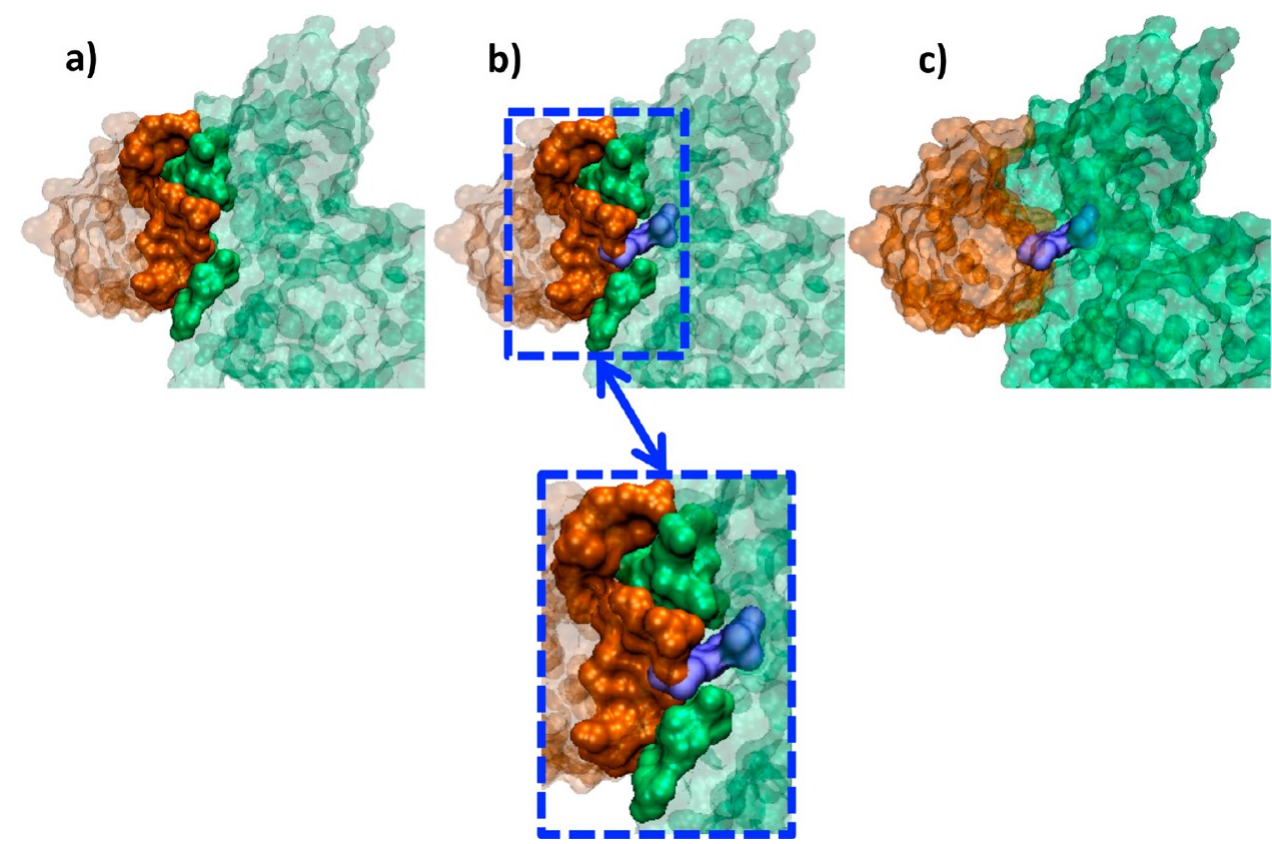

Figure 6. The CDK6 (green)-CDKN2D (orange) Complex and CHEBI: 792520 (purple) Interference. (a) The interface of CDKN2D-CDK6 is from PDB ID: 1BLX. (b,c) In the PDB, CHEBI: 792520 has a 3D structure in complex with CDK6 (PDB ID: 3NUX). When CDK6 proteins of 3NUX and 1BLX are superimposed, CHEBI: 792520 interferes with the CDK6 and CDKN2D interface. These two figures are predicted outcomes; no structural data are available.

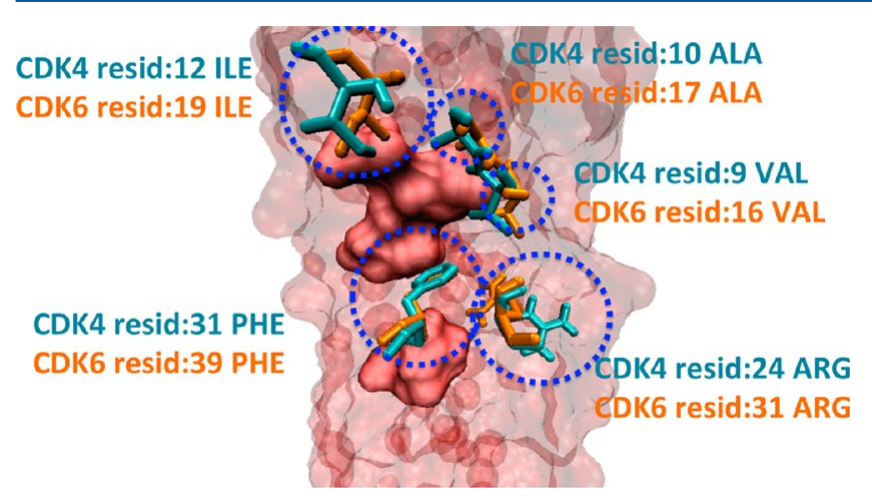

Figure 7. Hotspots of CDK4-CDKN2D and CDK6-CDKN2D Interfaces. The predicted hotspots of CDK4 (cyan) and CDK6 (orange) proteins are represented with "licorice" and the hotspots of CDKN2D are drawn as a red surface using VMD. ${ }^{90}$ The red, transparent body in the background is also CDKN2D protein. CDK4 and CDK6 have a number of identical hotspots when their interfaces with CDKN2D are superimposed.

designed to inhibit all the interactions of a single protein, one can simply remove this node from the network and investigate the changes. For making an accurate functional analysis, we need all known protein interactions in the p53 pathway. We constructed a p53 network that, regardless of the structural availability, contains all known protein interactions and proteins. We simulated the changes in the network when subject to node and interface attacks. We partitioned the network using the "Affinity Propagation" algorithm. ${ }^{94}$ This clustering algorithm determines the representative examples (examplars) of the graph and then partitions the network according to these examplars.

We mapped the experimentally validated PRISM interface predictions of the p53 pathway on Kohn's $\mathrm{MIM}^{70}$ as the starting point for constructing an experimentally validated network of protein interactions enriched with interfaces. We obtained a p53 PIN with 109 nodes and 227 edges. We expanded this network with the 66 PRISM predicted interfaces that were experimentally validated (26 interactions present in Kohn's MIM, 33 additional interactions from various experimental databases). We gathered a network of 115 nodes and 269 edges. Recall that there were a number of proteins from databases other than Kohn's MIM in our PRISM target. As a result, the number of nodes also increased (Figure 10). The clusters generated by the Affinity Propagation algorithm are shown using pie charts (Figure 11, top row). Clusters are named according to the highest degree node of that partition.

When the 1 jsuBC interface (template interface is between the CCNA2 and CDKN1B proteins) is attacked, 11 edges are removed from the network. Six of these are around the CDKN1B node. Therefore, this node is completely removed from the network by the 1 jsuBC interface attack in addition to the removal of 5 edges around other nodes. One can see that this attack causes the cluster with the RB1 hub node to get significantly bigger (refer to the slices of RB1cluster in the top and middle rows of Figure 11). RB1 now has a greater influence on the network. MYC is no more the hub node of a cluster (red slice present in the top row of Figure 11 disappears in the middle row of Figure 11), and the cluster of CDK2 enlarges from $9 \%$ of the nodes of network to $16 \%$ (Figure 11, middle row). A complete node attack targeting the CDKN1B protein means breaking all of this node's interactions detaching it from the network. PRISM predicts that all six interactions of CDKN1B have a similar structure to the 1 jsuBC interface. Thus, to block all of the interactions of CDKN1B, a drug has to attack the 1 jsuBC interface, which affects five more edges in the network. However, in the case of complete node attack on CDKN1B, only edges of this node are discarded from the network. We do not observe a significant change in the sizes of 

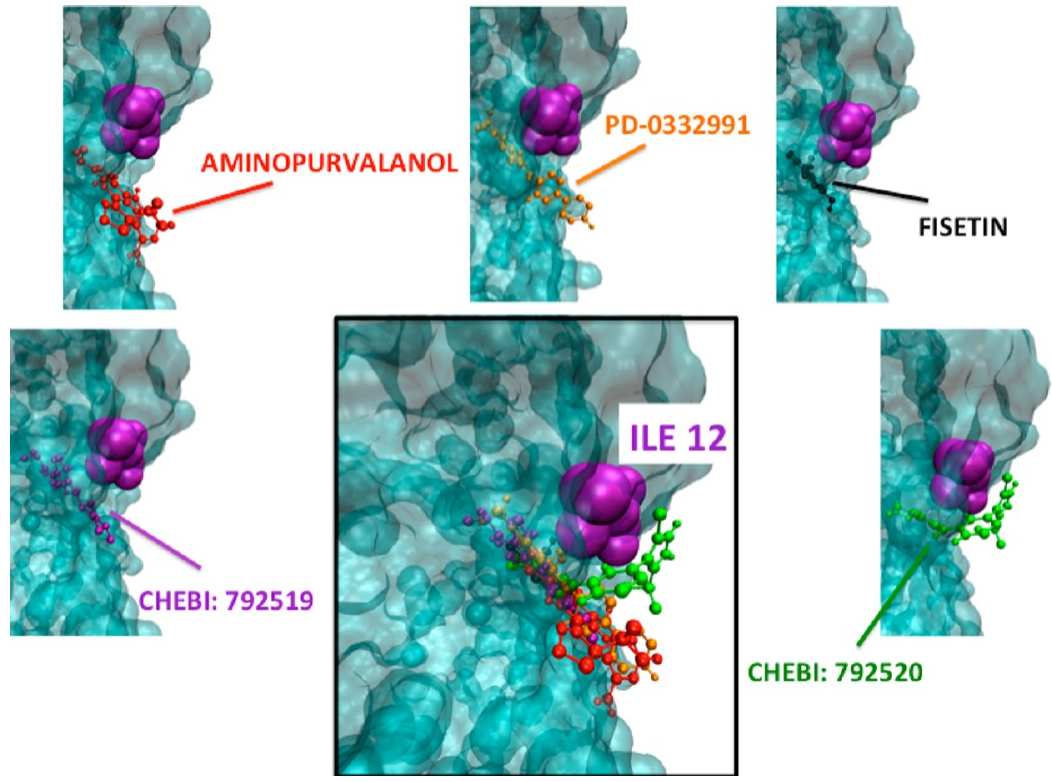

CHEBI: 792520

Figure 8. CDK4 Docking Simulations. AutoDock ${ }^{91}$ is used to dock the drugs (Aminopurvalanol, PD-0332991, CHEBI: 792519, CHEBI: 792520, and Fisetin) to candidate off-target CDK4. The hotspot (CDK6 residue Ile19) that is closest to the ligands' binding region on CDK6 is also present on the binding region of CDK4 (residue Ile12).
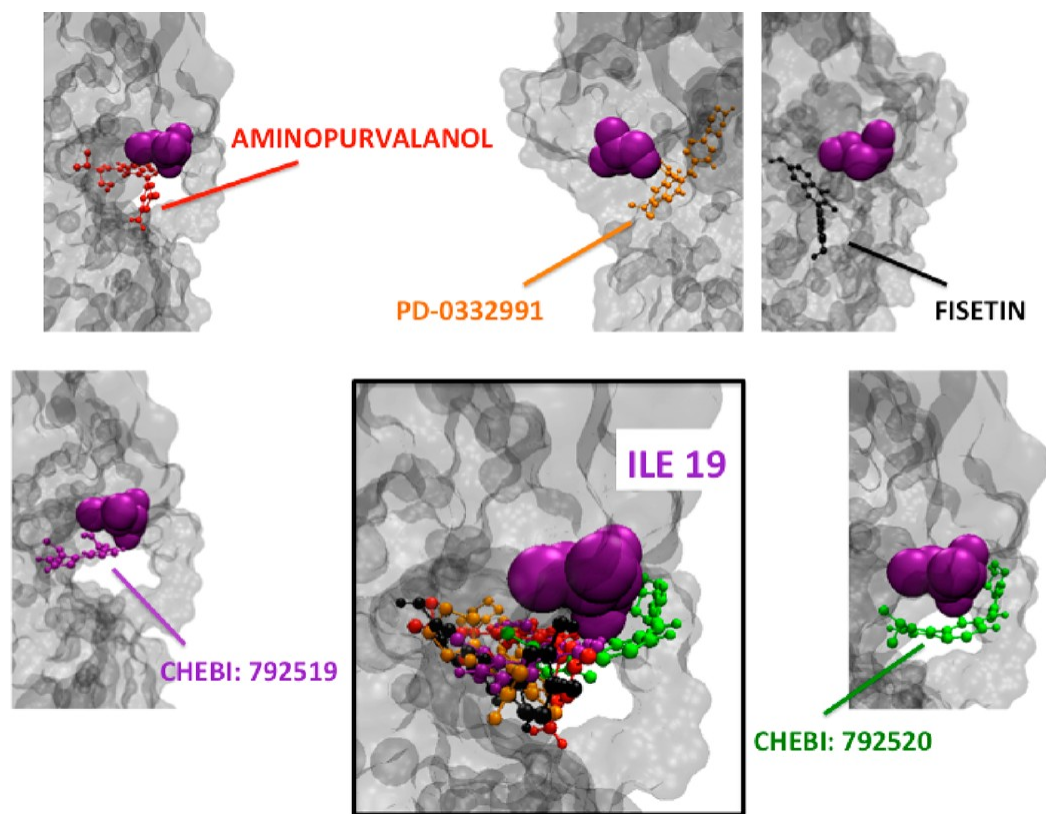

CHEBI: 792520

Figure 9. CDK6 Docking Simulations. AutoDock ${ }^{91}$ is used to dock the mentioned drugs (Aminopurvalanol, PD-0332991, CHEBI: 792519, CHEBI: 792520, and Fisetin) to primary target CDK6. The hotspot (CDK6 residue Ile19) that is closest to the ligands' binding region on CDK6 is also present on the binding region of CDK4 (residue 12).

\section{Table 2. AutoDock ${ }^{91}$ Results $^{a}$.}

$\begin{array}{cccccc} & \text { PD-0332991 } & \text { Fisetin } & \text { Aminopurvalanol } & \text { CHEBI: 792520 } & \text { CHEBI: 792519 } \\ \mathrm{CDK} 4 & -8.22 \mathrm{kcal} / \mathrm{mol} & -7.59 \mathrm{kcal} / \mathrm{mol} & -5.97 \mathrm{kcal} / \mathrm{mol} & -7.55 \mathrm{kcal} / \mathrm{mol} & -6.51 \mathrm{kcal} / \mathrm{mol} \\ \mathrm{CDK} 6 & -8.05 \mathrm{kcal} / \mathrm{mol} & -6.75 \mathrm{kcal} / \mathrm{mol} & -7.69 \mathrm{kcal} / \mathrm{mol} & -6.81 \mathrm{kcal} / \mathrm{mol} & -6.18 \mathrm{kcal} / \mathrm{mol} \\ \mathrm{rmsd} & 0.57 \AA & 0.68 \AA & 0.89 \AA & 1.83 \AA & 1.92 \AA\end{array}$

${ }^{a}$ Results given in terms of the lowest binding energy of the largest conformational clusters are in the first two rows. The RMSD values of superimpositions of the best poses of each drug molecule docked to CDK4 compared to CDK6 are in the last row.

the clusters following complete node attack (see top and bottom rows of Figure 11).

The changes observed after the interface attack appear reasonable. During the 1 jsuBC interface attack, CDKN1B is removed from the network, the CDK2 cluster gets bigger, and the influence of this protein on other nodes increases. Because CDKN1B has inhibitory activity on some CDK2 complexes, ${ }^{95}$ this change is expected. Once MYC is not a hub in a cluster, the 


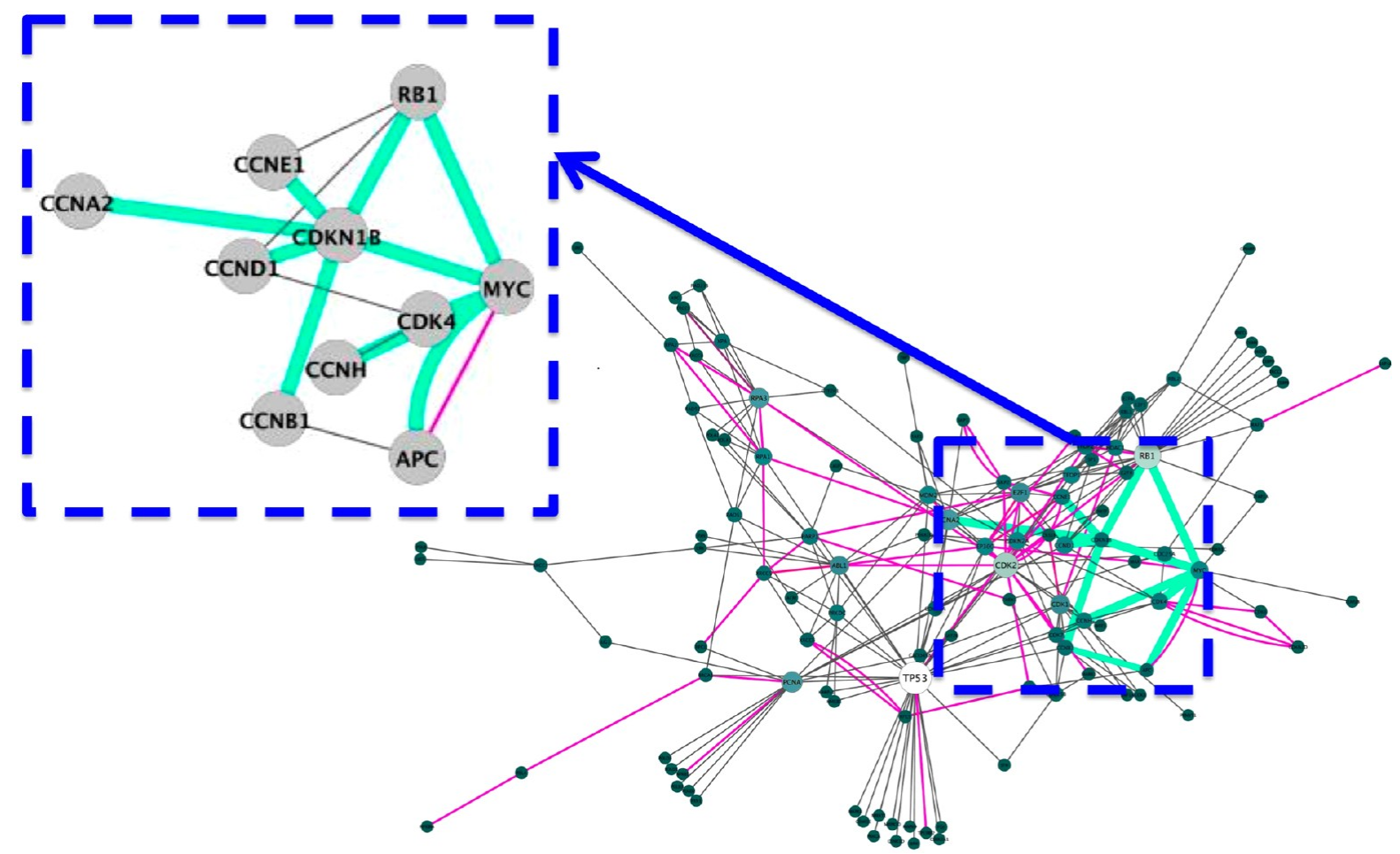

Figure 10. Structurally Enriched MIM Attacked Based on the 1jsuBC Interface. Experimentally validated edges of p53 P2IN mapped on the Kohn's MIM. ${ }^{70}$ The edges with interface structures are shown in pink, and the edges with 1 jsuBC interface is highlighted in green. In the close-up, figure edges with 1 jsuBC interfaces are also shown in green.

RB1 cluster expands. In the presence of MYC, the RB1 transcription is suppressed and MYC activates a set of miRNAs, which in turn inhibit the translation of $\mathrm{RB} 1 .^{96}$ Finally, CDKN1B and RB1 are tumor-suppressors. The RB1 cluster gets bigger when CDKN1B loses all of its interactions, possibly suggesting that RB1 may be involved in an alternative pathway.

Network Attack Scenarios Applied to P53 P2IN and Changes in the Network Robustness. The robustness of a network relates to its ability to withstand the damage caused by attacks. It can be expressed by topological parameters. The most commonly used robustness parameters are the average inverse geodesic length (AIGL) $)^{59,97}$ and the giant component $\operatorname{size}^{59}$ (GCS). To monitor the change in the connectedness of the nodes in the system, we use both.

For the p53 P2IN survivability analysis, several attack types and target selection strategies are used. These attack scenarios refer to partial or complete knockout of hub nodes and deletion of multiple edges that are scattered around the network. At each step, a new target is hit, and the topological parameters are recalculated until the network is left without interactions.

i. Hub Node Attack. A hub is the highest degree node of the network; it is the node that has the largest number of interactions. This attack type targets the largest degree node of the network. Hitting this element also affects its interacting partners and causes a serious disturbance in the network communication.

ii. Frequent Interface Attack. In P2IN, the number of occurrences of each interface type is known. In this strategy the most frequently observed interface is selected as the target of interface attack.

iii. Maximal Damage Strategy. The maximal damage strategy is a greedy algorithm, which was studied by Agoston et al. $^{25}$ It hits the component that will harm the network the most in each attack. This tactic may be used in both node and edge attack types. Removing multiple edges that are selected according to the maximal damage target selection strategy is a kind of a distributed attack. It targets the node or interface that is expected to cause the greatest possible harm.

Frequent Interface Attack Is as Harmful as Complete Hub Knockout, and It Is a More Realistic Scenario. Breaking an edge can be considered as the graphical representation of a drug blocking the interaction of two proteins. If we were to map node-targeted attacks (complete or partial knockout) to a drug mechanism, it would be a "magic bullet"; even if a drug would specifically bind to one protein, in most of the cases it may not obstruct all of its interactions. It seems that complete knockout is rarely observed in realistic drug action. The common "similar binding sites should recognize similar ligands" strategy, ${ }^{22}$ motivated us to develop the interface attack.

Complete/partial hub node attacks and interface attacks based on their frequencies of occurrence are performed on the p53 P2IN. In Figure 12, the change in the network robustness is plotted according to AIGL and GCS. The $x$-axis stands for the number of attacks, while the $y$-axis is the AIGL or GCS values during the attacks. A drop in AIGL or GCS of the network correlates with the damage caused to the system. The plots show that attacking the most frequent interface in the p53 signaling network is at least as harmful as complete removal of the hub nodes from the network. Thus, rather than targeting a well-connected protein, which is more likely to be essential, ${ }^{60}$ we may target edges that have similar interface structures.

The most frequent interface (PDB ID: 1JSU, chains: $B, C$ ) is observed 46 times in the p53 network. Twenty-one of these predictions are validated experimentally or present in the STRING database. If there was a drug designed to disturb one 


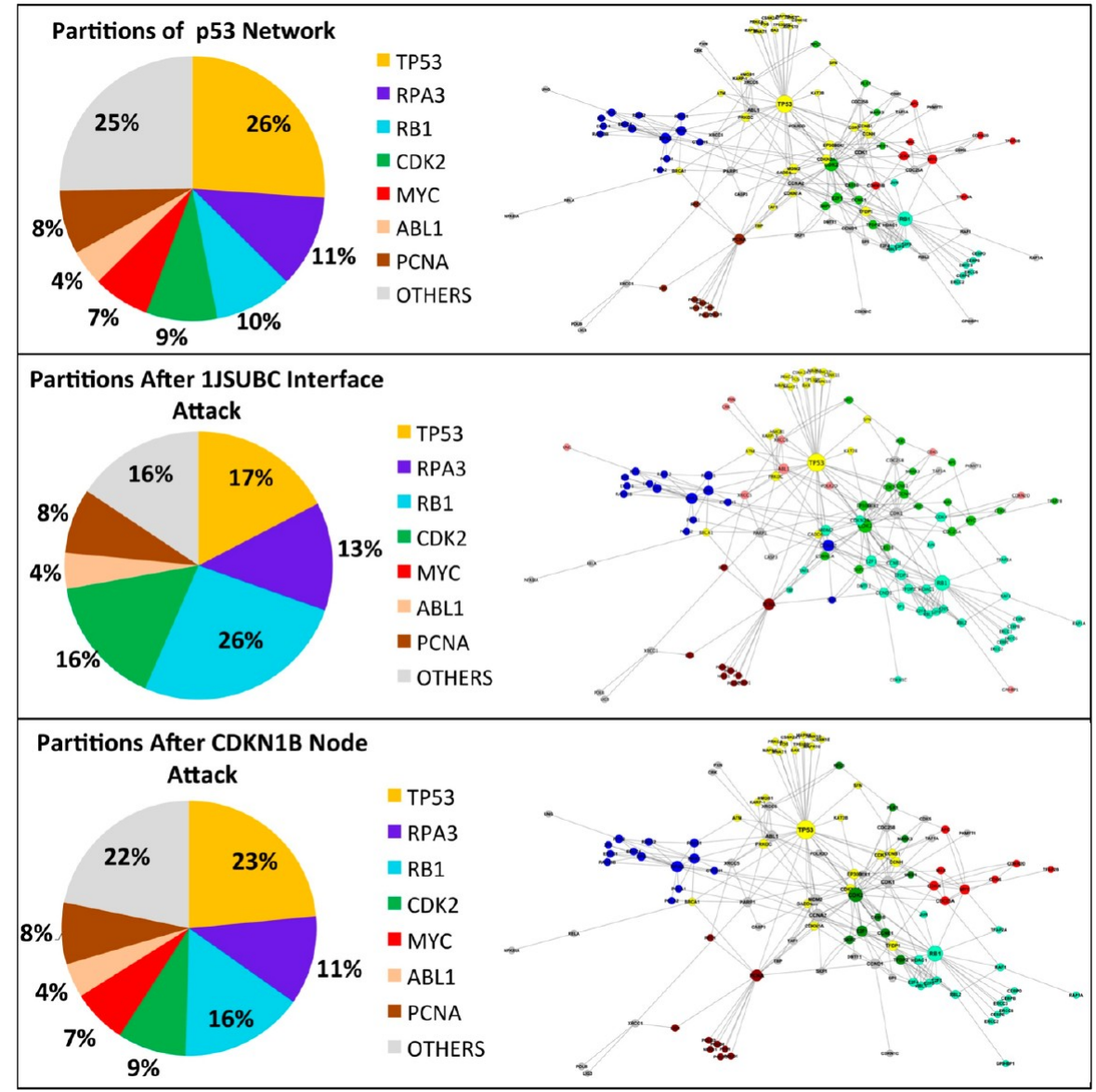

Figure 11. Pie Charts of Clusters Generated with Affinity Propagation Algorithm. In the pie charts, each slice represents a cluster, and they are named with the clusters' hub nodes. Percentages of the slices are the ratio of the node number in the corresponding cluster to the total number of nodes in the network. (Top row) Clusters of the network generated by mapping the experimentally validated PRISM predictions of p53 pathway onto Kohn's MIM. (Middle row) Clusters after 1jsuBC interface attack. (Bottom Row) Clusters after CDKN1B node attack.
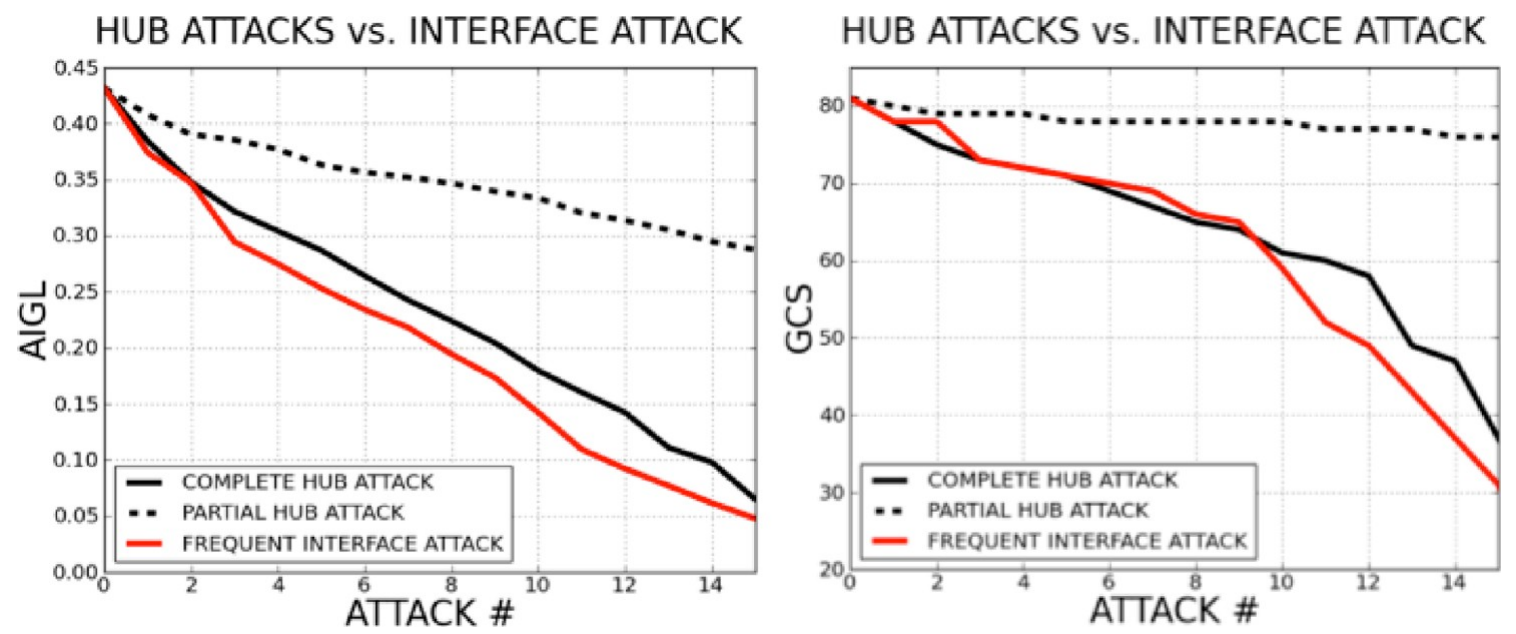

Figure 12. Hub Node Attack vs Most Frequent Interface Attack. The figure plots of the damage to the network following 15 successive complete hub node attacks, partial hub node attacks, and frequent interface attacks (for AIGL (left) and GCS (right) topological parameters). The results suggest that the most frequent interface attack and complete hub knockout lead to roughly the same damage, while the effect of the partial hub knockout is to a lesser extent.

of these 46 interactions, not just that particular edge, all 46 interactions could be hit. Hence, building the interface and interaction network of a biological system may be helpful for drug development.

\section{Interface Attack Is Not as Harmful as Distributed} Attack when Maximal Damage Strategy Is Applied. Agoston et al. $^{25}$ showed that rather than removing a node completely from the network, one could inflict similar damage 

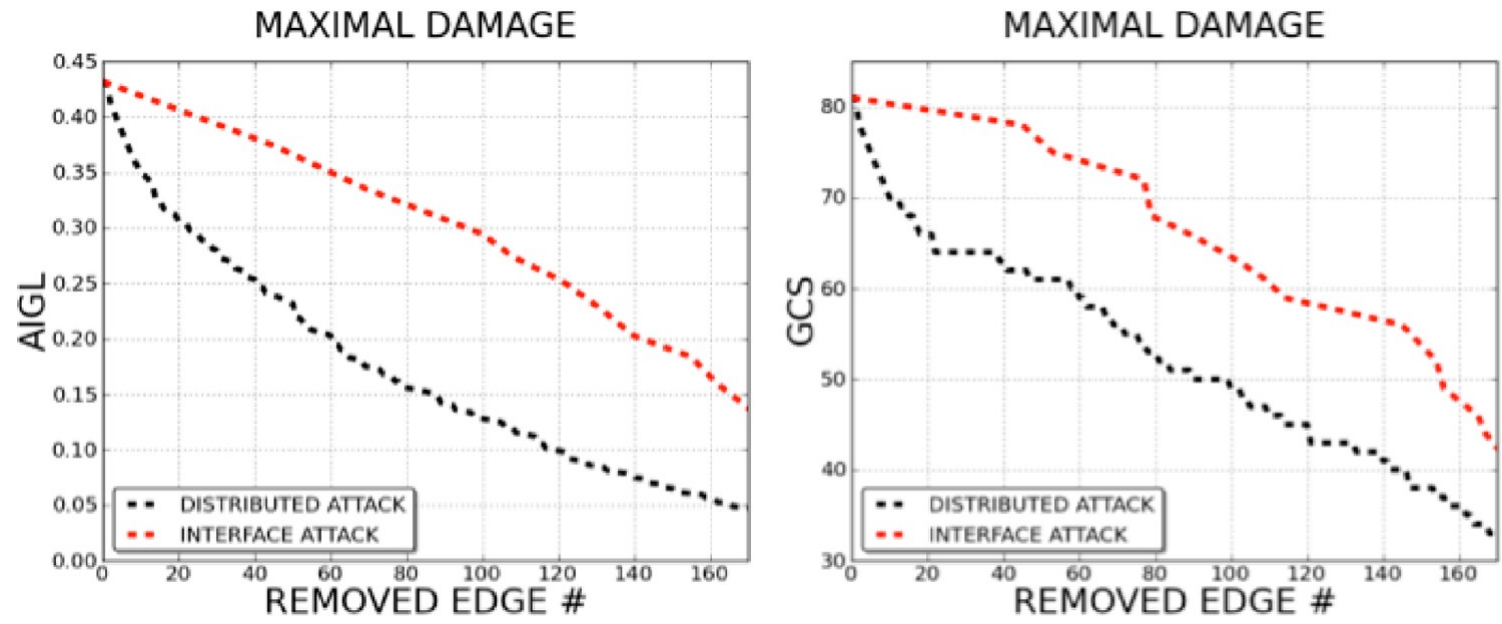

Figure 13. Maximal Successive Damage Strategy on Distributed and Interface Attack. Damage in the network (both according to AIGL (left) and GCS (right) topological parameters) is monitored under successive attacks. Distributed attack and interface attack are executed using the maximal damage strategy. The number of edges removed from the network in each attack is parallel to the harm attacks cause on the network. It is obvious that distributed attack is the most harmful strategy.
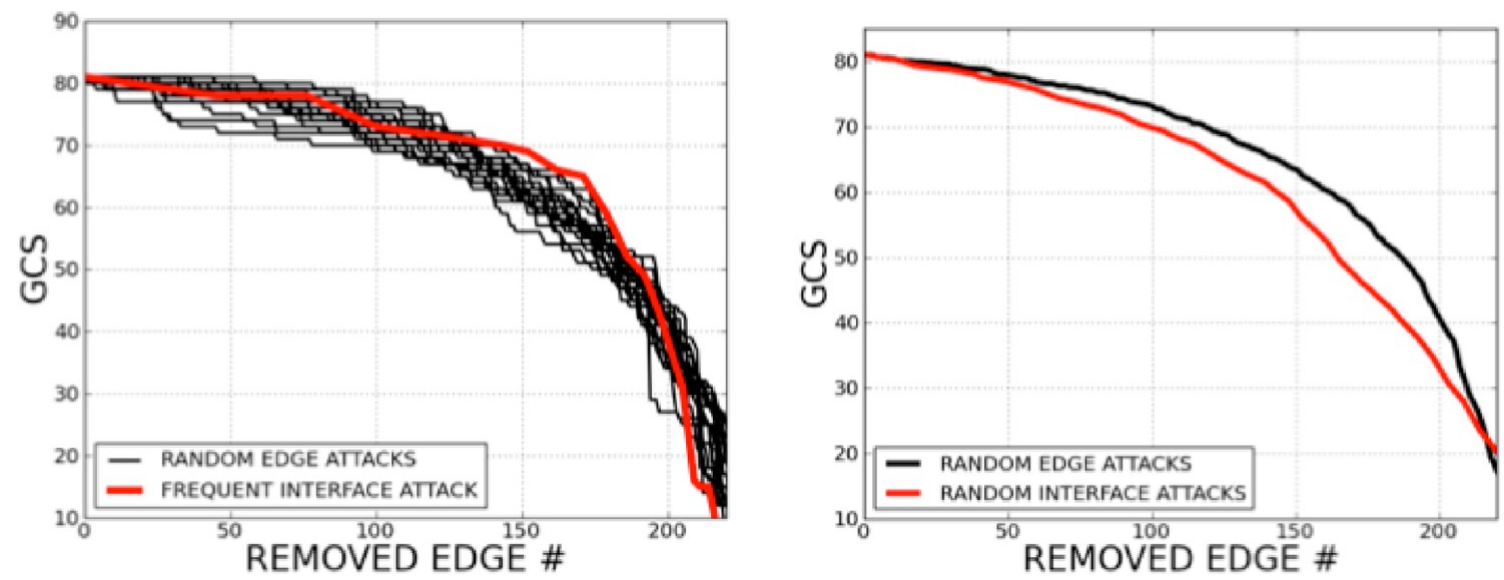

Figure 14. Random Edge Attacks vs Interface Attacks. The most frequent interface attack is relatively less harmful to the p53 P2IN than random edge attacks (left). However, the average of random interface attacks harms the network more than the average of random edge attacks (right). Consequently, random interface attacks give more harm to the network than frequent interface attacks.

by removing a number of edges distributed around the network. They chose the most destructive edges.

Interface attack is a kind of distributed attack, but it chooses the target edge set on the basis of interface similarity. We performed distributed attacks and interface attacks on the p53 P2IN. In this experiment, we followed a maximal damage target selection strategy by selecting the most damaging edges (distributed attack) or the most damaging interface in successive attacks. The comparison of the damage caused by distributed attack and interface attack is plotted in Figure 13. The $x$-axis is the number of edges removed during attacks, and the $y$-axis is the change in the network GCS and AIGL. It is clear that distributed attack harms the network more than interface attack. However, comparison of interface attack and distributed attack is not straightforward because distributed attack selects edges one by one, while interface attack chooses between sets of edges. This is why distributed attack is so harmful and is nearly the optimal attack strategy for collapsing the network. However, interface attack seems to be physically more suitable for simulating the impact of multi-target drugs on the network because the interactions affected by multi-target drugs are not always the most harmful.
Frequent Interfaces Are Not Observed on Topologically Critical Interactions. P53 P2IN is a small subnetwork, and it does not have a scale-free architecture. When random edge attacks are compared with frequent interface attacks (Figure 14) according to the change in giant component sizes, the most frequent interface attack is less harmful to the p53 P2IN than random edge attacks.

However, when the attacks are performed on randomly selected interfaces, we observe that on average they harm the network more than random edge attacks. Consequently, random interface attacks are more harmful to the network than frequent interface attacks; that is, a frequent interface is less likely to hit topologically critical elements of the network. This makes the network more resistant to failures.

\section{CONCLUSIONS}

We proposed a new network representation (P2IN) that introduces the structures of protein interfaces into the protein interaction networks (PINs). In addition to providing the binary information of whether two proteins interact with each other, the protein interface and interaction network (P2IN) also provides information on the structure of the complex that 
they form. This representation allows us to propose a new attack strategy of hitting edges between protein pairs that interact via structurally similar interfaces rather than nodes.

We generated the signaling network of the p53 P2IN and tested its robustness to various attacks. Both node and edge attacks are performed. The interface attack is found to be as destructive as hub node attacks; however, it is not as harmful as distributed attack that targets maximal edges. A drug that disturbs a frequent interface type may be as destructive as a drug targeting a high degree protein, suggesting the usefulness of considering the frequency of interface motifs during drug development. We discovered that some drugs (Aminopurvalanol, PD-0332991, CHEBI:792519, CHEBI:792520, and Fisetin) binding to CDK6 disrupt its interaction with CDKN2D. We applied our interface attack strategy to this case and found that drugs blocking this interface may also affect the interaction between CDK4 and CDKN2D. CDK4 also appears an off-target for drugs binding to CDK6. This example illustrates the promise in our strategy as a first step in identifying potential off-target drug hits. Finally, we provided a case study of a comparison between node and interface attacks. Challenging next steps are accounting for molecular flexibility. Proteins are highly dynamic, and structure-based drug discovery requires detailed structural treatment to uncover transient pockets that are unlikely to be observed in the static crystal snapshots and rigid docking. Nonetheless, systems-wide outcome involving possible off-targets of a drug is an important consideration and eventually would need to be integrated with detailed structural investigation in attempts to forecast potential side effects. Here, our concept of interface attack exploits structural motifs. It is inspired by network pharmacology, an emerging paradigm in drug discovery.

\section{MATERIALS AND METHODS}

Constructing Protein Interface and Interaction Network (P2IN). The first step of building a P2IN is to gather raw data of protein interactions and their 3D structures. Protein interactions are collected from the literature and databases; the 3D structure of the interfaces is obtained from application of PRISM. ${ }^{68,69}$ There may be more than one possible template interface for one interaction pair; in such a case, there is more than one possible binding site between two proteins. All possibilities are considered, and every matching interface template is included in the interface and interaction networks. Proteins whose interface sites cannot be predicted by the PRISM server are discarded. This decreases the number of proteins and interactions.

PRISM predicts interfaces for two target proteins that have $3 \mathrm{D}$ structures in the PDB. However, the interaction data gathered from the literature are UniProt ${ }^{98}$ IDs. Thus, target (UniProt) proteins should be mapped to PDB IDs, and these will constitute the input for PRISM. The main problem in this phase is that a protein may have multiple PDB IDs or its 3D structure might not be known. To overcome this inconsistency, proteins that do not have matching PDB chains are discarded, and all PDB counterparts of a protein are taken into account while constructing the network.

P53 P2IN. We studied the interactions between the proteins in the p53 signaling pathway. The list of proteins that are involved in this pathway was compiled from the literature ${ }^{70}$ and databases by Tuncbag et al. ${ }^{50}$ Among these proteins, 85 had 3D structures in the PDB. The interaction and interface data is obtained from PRISM predictions. We used 1037 template interfaces that were extracted from the $\mathrm{PDB}^{99}$ for the prediction process. The resulting interface predictions with energies lower than -10 are accepted.

PRISM predicted 251 interactions among 81 proteins, and there are 46 different interface structures in the network. The number of proteins dropped from 85 to 81 because PRISM did not infer interactions for some proteins. If we were to link each protein in the network to other proteins, we would end up with \%300 edges. PRISM infers 251 interactions out of those 3300 possibilities, and $41 \%$ of those predictions are already known. Furthermore, in the generated p53 P2IN, there are 15 PPIs that have PDB structures in complex form. PRISM was able to predict 13 of those interfaces correctly (Table SI2, Supporting Information).

Docking Parameters. For adding polar hydrogens, assigning Gasteiger charges and drawing grid boxes AutoDockTools 1.5.4 ${ }^{91}$ was used. Binding affinities were calculated with AutoGrid version 4. Lamarckian genetic algorithm (trials of 50 dockings, population size of 150 , and maximum number of generations of 27000) was used to do the docking experiments using AutoDock 4.0.91

Clustering Algorithm. We partitioned the network according to the "Affinity Propagation" algorithm ${ }^{94}$ with the help of Clustermaker plugin ${ }^{100}$ of Cytoscape. ${ }^{101}$

Mapping the Experimentally Validated PRISM Interface Predictions of p53 Pathway on the Kohn's MIM. Kohn's MIM has some nodes that do not have a protein counterpart or some nodes correspond to multiple proteins. Before constructing the PIN, we updated nodes in Kohn's MIM by removing or expanding some of them (Table SI3, Supporting Information). If a node was replaced with multiple proteins, the number of interactions automatically increased. We searched the String database for validating the new edges and picked the ones that were coming from experiments or databases. For example, the "CDK4-6" node corresponds to three proteins (CDK4, CDK5, CDK6). In the original map there was an interaction between "CDK7" and "CDK4-6". The "CDK7" interactions with CDK4 and CDK5 are validated but not with CDK6. The full list of interactions can be found in Table SI4 of the Supporting Information.

Robustness Measures. AIGL is the sum of the inverses of all shortest paths, divided by the number of possible node combinations. The definition is given in eq 1 . The notation used is as follows: $l=$ average geodesic length; $n=$ number of nodes; $i, j=$ proteins; and $d_{i j}=$ distance between proteins $i$ and $j$.

If there is no path connecting nodes $i$ and $j$, the distance between them is set to infinity. Some studies use the average geodesic length, but we preferred to use AIGL. Even after several attacks, AIGL will not be equal to infinity because if there is no navigable route between $i$ and $j, 1 /\left(d_{i j}\right)=0$.

$$
l^{-1}=\frac{1}{(n)(n-1)} \sum_{i \neq j} \frac{1}{d_{i j}}
$$

GCS is the number of nodes in the network's largest connected subgraph, and it may give important clues about the collapsing mechanism of network under attacks.

\section{ASSOCIATED CONTENT}

\section{S Supporting Information}

Table SI1 provides PRISM interaction predictions for p53 network. Table SI2 shows the correctness status of PRISM 
predictions for 15 interactions that have available PDB structures. A full list of proteins in Kohn's MIM can be found in Table SI3. In Table SI4, the list of updated interactions of Kohn's MIM is given. Figure SI1 highlights the CDK6 binding site (one chain of the CDK6-CDKN2D interface) on CDK6-drug complexes present in PDB, and shows the overlap between the drugs and the binding site. Table SI5 contains the rmsd values of CDK6 structures we used in this study. Figure SI 2 depicts the hotspots of CDK4 and the drugs docked on CDK4, while Figure SI3 depicts the hotspots of CDK6 and the drugs docked on CDK6. Figure SI4 provides the superimposition of pockets of CDK6 (cyan) and CDK4 (dark blue) using VMD visualization tool. Figure SI5 shows the change in drugs' conformations when the pockets of CDK6 (dark blue) and CDK4 (red) are superimposed. This material is available free of charge via the Internet at http://pubs.acs.org.

\section{AUTHOR INFORMATION}

\section{Corresponding Author}

*E-mail: agursoy@ku.edu.tr

\section{Notes}

The authors declare no competing financial interest.

\section{ACKNOWLEDGMENTS}

This work is supported by TUBITAK (Research Grant Numbers 109T343 and 109E207) and Turkish Academy of Sciences (TUBA). This project is funded in whole or in part with federal funds from the National Cancer Institute, National Institutes of Health, under contract number HHSN261200800001E. The content of this publication does not necessarily reflect the views or policies of the Department of Health and Human Services, nor does mention of trade names, commercial products, or organizations imply endorsement by the U.S. Government. This research is supported (in part) by the Intramural Research Program of the NIH, National Cancer Institute, Center for Cancer Research.

\section{REFERENCES}

(1) Xie, L.; Bourne, P. E. Structure-based systems biology for analyzing off-target binding. Curr. Opin. Struct. Biol. 2011, 21, 189199.

(2) Mestres, J.; Gregori-Puigjane, E.; Valverde, S.; Sole, R. V. Data completeness: The Achilles heel of drug-target networks. Nat. Biotechnol. 2008, 26, 983-984.

(3) Cohen, A. A.; Geva-Zatorsky, N.; Eden, E.; Frenkel-Morgenstern, M.; Issaeva, I.; Sigal, A.; Milo, R.; Cohen-Saidon, C.; Liron, Y.; Kam, Z.; Cohen, L.; Danon, T.; Perzov, N.; Alon, U. Dynamic proteomics of individual cancer cells in response to a drug. Science 2008, 322, 15111516.

(4) Pujol, A.; Mosca, R.; Farres, J.; Aloy, P. Unveiling the role of network and systems biology in drug discovery. Trends Pharmacol. Sci. 2010, 31, 115-123.

(5) Cheng, A. C.; Coleman, R. G.; Smyth, K. T.; Cao, Q.; Soulard, P.; Caffrey, D. R.; Salzberg, A. C.; Huang, E. S. Structure-based maximal affinity model predicts small-molecule druggability. Nat. Biotechnol. 2007, 25, 71-75.

(6) Rarey, M.; Kramer, B.; Lengauer, T.; Klebe, G. A fast flexible docking method using an incremental construction algorithm. J. Mol. Biol. 1996, 261, 470-489.

(7) Liu, X.; Ouyang, S.; Yu, B.; Liu, Y.; Huang, K.; Gong, J.; Zheng, S.; Li, Z.; Li, H.; Jiang, H. PharmMapper server: A web server for potential drug target identification using pharmacophore mapping approach. Nucleic Acids Res. 2010, 38, W609-W614.

(8) Byvatov, E.; Fechner, U.; Sadowski, J.; Schneider, G. Comparison of support vector machine and artificial neural network systems for drug/nondrug classification. J. Chem. Inf. Comput. Sci. 2003, 43, 18821889.

(9) Keiser, M. J.; Roth, B. L.; Armbruster, B. N.; Ernsberger, P.; Irwin, J. J.; Shoichet, B. K. Relating protein pharmacology by ligand chemistry. Nat. Biotechnol. 2007, 25, 197-206.

(10) Campillos, M.; Kuhn, M.; Gavin, A. C.; Jensen, L. J.; Bork, P. Drug target identification using side-effect similarity. Science 2008, 321, 263-266.

(11) Scheiber, J.; Jenkins, J. L.; Sukuru, S. C.; Bender, A.; Mikhailov, D.; Milik, M.; Azzaoui, K.; Whitebread, S.; Hamon, J.; Urban, L.; Glick, M.; Davies, J. W. Mapping adverse drug reactions in chemical space. J. Med. Chem. 2009, 52, 3103-3107.

(12) Laurie, A. T.; Jackson, R. M. Q-SiteFinder: An energy-based method for the prediction of protein-ligand binding sites. Bioinformatics 2005, 21, 1908-1916.

(13) Huang, B.; Schroeder, M. LIGSITEcsc: Predicting ligand binding sites using the Connolly surface and degree of conservation. BMC Struct. Biol. 2006, 6, 19.

(14) Glaser, F.; Morris, R. J.; Najmanovich, R. J.; Laskowski, R. A.; Thornton, J. M. A method for localizing ligand binding pockets in protein structures. Proteins 2006, 62, 479-488.

(15) Weisel, M.; Proschak, E.; Schneider, G. PocketPicker: Analysis of ligand binding-sites with shape descriptors. Chem. Cent. J. 2007, 1, 7.

(16) Laskowski, R. A. SURFNET: A program for visualizing molecular surfaces, cavities, and intermolecular interactions. J. Mol. Graph. 1995, 13, 323-30, 307-308.

(17) Xie, L.; Bourne, P. E. A robust and efficient algorithm for the shape description of protein structures and its application in predicting ligand binding sites. BMC Bioinf. 2007, 8 (Suppl 4), S9.

(18) Zhu, S.; Okuno, Y.; Tsujimoto, G.; Mamitsuka, H. A probabilistic model for mining implicit 'chemical compound-gene' relations from literature. Bioinformatics 2005, 21 (Suppl 2), ii245ii251.

(19) Hansen, N. T.; Brunak, S.; Altman, R. B. Generating genomescale candidate gene lists for pharmacogenomics. Clin. Pharmacol. Ther. 2009, 86, 183-189.

(20) Yamanishi, Y.; Araki, M.; Gutteridge, A.; Honda, W.; Kanehisa, M. Prediction of drug-target interaction networks from the integration of chemical and genomic spaces. Bioinformatics 2008, 24, i232-i240.

(21) Yamanishi, Y.; Kotera, M.; Kanehisa, M.; Goto, S. Drug-target interaction prediction from chemical, genomic and pharmacological data in an integrated framework. Bioinformatics 2010, 26, i246-i254.

(22) Defranchi, E.; Schalon, C.; Messa, M.; Onofri, F.; Benfenati, F.; Rognan, D. Binding of protein kinase inhibitors to synapsin I inferred from pair-wise binding site similarity measurements. PLoS One 2010, 5, e12214.

(23) Badis, G.; Berger, M. F.; Philippakis, A. A.; Talukder, S.; Gehrke, A. R.; Jaeger, S. A.; Chan, E. T.; Metzler, G.; Vedenko, A.; Chen, X.; Kuznetsov, H.; Wang, C. F.; Coburn, D.; Newburger, D. E.; Morris, Q.; Hughes, T. R.; Bulyk, M. L. Diversity and complexity in DNA recognition by transcription factors. Science 2009, 324, 1720-1723.

(24) Roy, J.; Cyert, M. S. Cracking the phosphatase code: Docking interactions determine substrate specificity. Sci. Signal 2009, 2, re9.

(25) Agoston, V.; Csermely, P.; Pongor, S. Multiple weak hits confuse complex systems: A transcriptional regulatory network as an example. Phys. Rev. E: Stat., Nonlinear, Soft Matter Phys. 2005, 71, 051909.

(26) Keskin, O.; Gursoy, A.; Ma, B.; Nussinov, R. Principles of protein-protein interactions: What are the preferred ways for proteins to interact? Chem. Rev. 2008, 108, 1225-1244.

(27) Arkin, M. R.; Wells, J. A. Small-molecule inhibitors of proteinprotein interactions: Progressing towards the dream. Nat. Rev. Drug Discovery 2004, 3, 301-317.

(28) Chene, P. Drugs targeting protein-protein interactions. ChemMedChem 2006, 1, 400-411.

(29) Arkin, M. R.; Whitty, A. The road less traveled: Modulating signal transduction enzymes by inhibiting their protein-protein interactions. Curr. Opin. Chem. Biol. 2009, 13, 284-290. 
(30) Gao, M.; Skolnick, J. Structural space of protein-protein interfaces is degenerate, close to complete, and highly connected. Proc. Natl. Acad. Sci. U.S.A. 2010, 107, 22517-22522.

(31) Fry, D. C.; Vassilev, L. T. Targeting protein-protein interactions for cancer therapy. J. Mol. Med. (Berlin) 2005, 83, 955963.

(32) Wells, J. A.; McClendon, C. L. Reaching for high-hanging fruit in drug discovery at protein-protein interfaces. Nature 2007, 450, 1001-1009.

(33) Whitty, A.; Kumaravel, G. Between a rock and a hard place? Nat. Chem. Biol. 2006, 2, 112-118.

(34) Fuller, J. C.; Burgoyne, N. J.; Jackson, R. M. Predicting druggable binding sites at the protein-protein interface. Drug Discovery Today 2009, 14, 155-161.

(35) Gonzalez-Ruiz, D.; Gohlke, H. Targeting protein-protein interactions with small molecules: Challenges and perspectives for computational binding epitope detection and ligand finding. Curr. Med. Chem. 2006, 13, 2607-2625.

(36) Wishart, D. S.; Knox, C.; Guo, A. C.; Cheng, D.; Shrivastava, S.; Tzur, D.; Gautam, B.; Hassanali, M. DrugBank: A knowledgebase for drugs, drug actions and drug targets. Nucleic Acids Res. 2008, 36, D901-D906.

(37) Kuritzkes, D.; Kar, S.; Kirkpatrick, P. Fresh from the pipeline; maraviroc. Nat. Rev. Drug Discovery 2008, 7, 15-16.

(38) Domling, A. Small molecular weight protein-protein interaction antagonists: An insurmountable challenge? Curr. Opin. Chem. Biol. 2008, 12, 281-291.

(39) Albert, R.; Jeong, H.; Barabasi, A. L. Error and attack tolerance of complex networks. Nature 2000, 406, 378-382.

(40) Barabasi, A. L.; Oltvai, Z. N. Network biology: Understanding the cell's functional organization. Nat. Rev. Genet. 2004, 5, 101-113.

(41) Gerdes, S. Y.; Scholle, M. D.; Campbell, J. W.; Balazsi, G.; Ravasz, E.; Daugherty, M. D.; Somera, A. L.; Kyrpides, N. C.; Anderson, I.; Gelfand, M. S.; Bhattacharya, A.; Kapatral, V.; D’Souza, M.; Baev, M. V.; Grechkin, Y.; Mseeh, F.; Fonstein, M. Y.; Overbeek, R.; Barabasi, A. L.; Oltvai, Z. N.; Osterman, A. L. Experimental determination and system level analysis of essential genes in Escherichia coli MG1655. J. Bacteriol. 2003, 185, 5673-5684.

(42) Kar, G.; Gursoy, A.; Keskin, O. Human cancer protein-protein interaction network: A structural perspective. PLoS Comput. Biol. 2009, 5, e1000601.

(43) Gursoy, A.; Keskin, O.; Nussinov, R. Topological properties of protein interaction networks from a structural perspective. Biochem. Soc. Trans. 2008, 36, 1398-1403.

(44) Keskin, O.; Nussinov, R. Similar binding sites and different partners: implications to shared proteins in cellular pathways. Structure 2007, 15, 341-354.

(45) Tuncbag, N.; Gursoy, A.; Guney, E.; Nussinov, R; Keskin, O. Architectures and functional coverage of protein-protein interfaces. $J$. Mol. Biol. 2008, 381, 785-802.

(46) Berman, H. M.; Westbrook, J.; Feng, Z.; Gilliland, G.; Bhat, T. N.; Weissig, H.; Shindyalov, I. N.; Bourne, P. E. The Protein Data Bank. Nucleic Acids Res. 2000, 28, 235-242.

(47) Shatsky, M.; Nussinov, R.; Wolfson, H. J. A method for simultaneous alignment of multiple protein structures. Proteins 2004, 56, $143-156$.

(48) Mashiach, E.; Nussinov, R.; Wolfson, H. J. FiberDock: A web server for flexible induced-fit backbone refinement in molecular docking. Nucleic Acids Res. 2010, 38, W457-W461.

(49) Mashiach, E.; Nussinov, R.; Wolfson, H. J. FiberDock: Flexible induced-fit backbone refinement in molecular docking. Proteins 2010, 78, 1503-1519.

(50) Tuncbag, N.; Keskin, O.; Nussinov, R.; Gursoy, A. Fast and accurate modeling of protein-protein interactions by combining template-interface-based docking with flexible refinement. Proteins 2012, 80, 1239-1249.

(51) Keskin, O.; Tsai, C. J.; Wolfson, H.; Nussinov, R. A new, structurally nonredundant, diverse data set of protein-protein interfaces and its implications. Protein Sci. 2004, 13, 1043-1055.
(52) Keskin, O.; Ma, B.; Rogale, K.; Gunasekaran, K.; Nussinov, R. Protein-protein interactions: Organization, cooperativity and mapping in a bottom-up systems biology approach. Phys. Biol. 2005, 2, S24-S35.

(53) Xie, L.; Li, J.; Bourne, P. E. Drug discovery using chemical systems biology: Identification of the protein-ligand binding network to explain the side effects of CETP inhibitors. PLoS Comput. Biol. 2009, 5, e1000387.

(54) Haupt, V. J.; Schroeder, M. Old friends in new guise: Repositioning of known drugs with structural bioinformatics. Brief Bioinform 2011, 12, 312-326.

(55) Perot, S.; Sperandio, O.; Miteva, M. A.; Camproux, A. C.; Villoutreix, B. O. Druggable pockets and binding site centric chemical space: A paradigm shift in drug discovery. Drug Discovery Today 2010, $15,656-667$.

(56) Hopkins, A. L. Network pharmacology: the next paradigm in drug discovery. Nat. Chem. Biol. 2008, 4, 682-690.

(57) P. Erdös, A. R. On random graphs. Publicationes Mathematicae 1959, 6, 290-297.

(58) Barabasi, A. L.; Albert, R. Emergence of scaling in random networks. Science 1999, 286, 509-512.

(59) Holme, P.; Kim, B. J.; Yoon, C. N.; Han, S. K. Attack vulnerability of complex networks. Phys. Rev. E: Stat., Nonlinear, Soft Matter Phys. 2002, 65, 056109.

(60) Jeong, H.; Mason, S. P.; Barabasi, A. L.; Oltvai, Z. N. Lethality and centrality in protein networks. Nature 2001, 411, 41-42.

(61) Dartnell, L.; Simeonidis, E.; Hubank, M.; Tsoka, S.; Bogle, I. D.; Papageorgiou, L. G. Robustness of the p53 network and biological hackers. FEBS Lett. 2005, 579, 3037-3042.

(62) Crucitti P, L. V.; Marchiori, M; Rapisard, A Efficiency of scalefree networks: Error and attack tolerance. Phys. A 2003, 622-642.

(63) Zhang, D. M.; Yin, Y. P.; Tan, J; Pan, G. J.; He, M. H. Multiple partial attacks on complex networks. Chin. Phys. Soc. 2008, 25, 769772.

(64) Harris, S. L.; Levine, A. J. The p53 pathway: Positive and negative feedback loops. Oncogene 2005, 24, 2899-2908.

(65) Hanahan, D.; Weinberg, R. A. The hallmarks of cancer. Cell 2000, 100, 57-70.

(66) Vogelstein, B.; Lane, D.; Levine, A. J. Surfing the p53 network. Nature 2000, 408, 307-310.

(67) Haupt, S.; Berger, M.; Goldberg, Z.; Haupt, Y. Apoptosis - the p53 network. J Cell Sci 2003, 116, 4077-4085.

(68) Tuncbag, N.; Gursoy, A.; Nussinov, R.; Keskin, O. Predicting protein-protein interactions on a proteome scale by matching evolutionary and structural similarities at interfaces using PRISM. Nat. Protoc. 2011, 6, 1341-1354.

(69) Ogmen, U.; Keskin, O.; Aytuna, A. S.; Nussinov, R.; Gursoy, A. PRISM: Protein interactions by structural matching. Nucleic Acids Res. 2005, 33, W331-W336.

(70) Kohn, K. W. Molecular interaction map of the mammalian cell cycle control and DNA repair systems. Molecular biology of the cell 1999, 10, 2703-2734.

(71) Prasad, T. S.; Kandasamy, K.; Pandey, A. Human protein reference database and human proteinpedia as discovery tools for systems biology. Methods Mol. Biol. 2009, 577, 67-79.

(72) Ceol, A.; Chatr Aryamontri, A.; Licata, L.; Peluso, D.; Briganti, L.; Perfetto, L.; Castagnoli, L.; Cesareni, G. MINT, the molecular interaction database: 2009 update. Nucleic Acids Res. 2010, 38, D532D539.

(73) Aranda, B.; Achuthan, P.; Alam-Faruque, Y.; Armean, I.; Bridge, A.; Derow, C.; Feuermann, M.; Ghanbarian, A. T.; Kerrien, S.; Khadake, J.; Kerssemakers, J.; Leroy, C.; Menden, M.; Michaut, M.; Montecchi-Palazzi, L.; Neuhauser, S. N.; Orchard, S.; Perreau, V.; Roechert, B.; van Eijk, K.; Hermjakob, H. The IntAct molecular interaction database in 2010. Nucleic Acids Res. 2010, 38, D525-D531.

(74) Matthews, L.; Gopinath, G.; Gillespie, M.; Caudy, M.; Croft, D.; de Bono, B.; Garapati, P.; Hemish, J.; Hermjakob, H.; Jassal, B.; Kanapin, A.; Lewis, S.; Mahajan, S.; May, B.; Schmidt, E.; Vastrik, I.; Wu, G.; Birney, E.; Stein, L.; D’Eustachio, P. Reactome knowledgebase 
of human biological pathways and processes. Nucleic Acids Res. 2009, 37, D619-D622.

(75) Stark, C.; Breitkreutz, B. J.; Reguly, T.; Boucher, L.; Breitkreutz, A.; Tyers, M. BioGRID: A general repository for interaction datasets. Nucleic Acids Res. 2006, 34, D535-D539.

(76) Cerami, E. G.; Gross, B. E.; Demir, E.; Rodchenkov, I.; Babur, O.; Anwar, N.; Schultz, N.; Bader, G. D.; Sander, C. Pathway Commons, a web resource for biological pathway data. Nucleic Acids Res. 2011, 39, D685-D690.

(77) Schaefer, C. F.; Anthony, K.; Krupa, S.; Buchoff, J.; Day, M.; Hannay, T.; Buetow, K. H. PID: The pathway interaction database. Nucleic Acids Res. 2009, 37, D674-D679.

(78) Szklarczyk, D.; Franceschini, A.; Kuhn, M.; Simonovic, M.; Roth, A.; Minguez, P.; Doerks, T.; Stark, M.; Muller, J.; Bork, P.; Jensen, L. J.; von Mering, C. The STRING database in 2011: Functional interaction networks of proteins, globally integrated and scored. Nucleic Acids Res. 2011, 39, D561-D568.

(79) Bolton, E, W. Y., Thiessen, P. A., Bryant, S. H. PubChem: Integrated Platform of Small Molecules and Biological Activities. In Annual Reports in Computational Chemistry; American Chemical Society: Washington, DC, 2008; Vol. 4.

(80) Lu, H.; Schulze-Gahmen, U. Toward understanding the structural basis of cyclin-dependent kinase 6 specific inhibition. J. Med. Chem. 2006, 49, 3826-3831.

(81) Baughn, L. B.; Di Liberto, M.; Wu, K.; Toogood, P. L.; Louie, T.; Gottschalk, R.; Niesvizky, R.; Cho, H.; Ely, S.; Moore, M. A.; Chen-Kiang, S. A novel orally active small molecule potently induces G1 arrest in primary myeloma cells and prevents tumor growth by specific inhibition of cyclin-dependent kinase 4/6. Cancer Res. 2006, 66, 7661-7667.

(82) Chen, X.; Ji, Z. L.; Chen, Y. Z. TTD: Therapeutic target database. Nucleic Acids Res. 2002, 30, 412-415.

(83) Cho YS, B. M.; Brain, C; Chen, C. H.; Cheng, H; Chopra, R; Chung, K; Groarke, J; He, G; Hou, Y; Kim, S; Kovats, S; Lu, Y; O’Reilly, M; Shen, J; Smith, T; Trakshel, G; Vögtle, M; Xu, M; Xu, M; Sung, M. J. 4-(Pyrazol-4-yl)-pyrimidines as selective inhibitors of cyclin-dependent kinase 4/6. J. Med. Chem. 2010, 53, 7938-7957.

(84) Lu, H.; Chang, D. J.; Baratte, B.; Meijer, L.; Schulze-Gahmen, U. Crystal structure of a human cyclin-dependent kinase 6 complex with a flavonol inhibitor, fisetin. J. Med. Chem. 2005, 48, 737-743.

(85) Magrane, M.; Consortium, U. UniProt knowledgebase: A hub of integrated protein data. Database (Oxford) 2011, 2011, bar009.

(86) Brotherton, D. H.; Dhanaraj, V.; Wick, S.; Brizuela, L.; Domaille, P. J.; Volyanik, E.; Xu, X.; Parisini, E.; Smith, B. O.; Archer, S. J.; Serrano, M.; Brenner, S. L.; Blundell, T. L.; Laue, E. D. Crystal structure of the complex of the cyclin D-dependent kinase Cdk6 bound to the cell-cycle inhibitor p19INK4d. Nature 1998, 395, 244250.

(87) Russo, A. A.; Tong, L.; Lee, J. O.; Jeffrey, P. D.; Pavletich, N. P. Structural basis for inhibition of the cyclin-dependent kinase Cdk6 by the tumour suppressor p16INK4a. Nature 1998, 395, 237-243.

(88) Chan, F. K.; Zhang, J.; Cheng, L.; Shapiro, D. N.; Winoto, A. Identification of human and mouse p19, a novel CDK4 and CDK6 inhibitor with homology to p16ink4. Mol. Cell. Biol. 1995, 15, 26822688.

(89) Tuncbag, N.; Gursoy, A.; Keskin, O. Identification of computational hot spots in protein interfaces: Combining solvent accessibility and inter-residue potentials improves the accuracy. Bioinformatics 2009, 25, 1513-1520.

(90) Humphrey, W.; Dalke, A.; Schulten, K. VMD: Visual molecular dynamics. J. Mol. Graph. 1996, 14, 33-8, 27-28.

(91) Morris, G. M.; Huey, R.; Lindstrom, W.; Sanner, M. F.; Belew, R. K.; Goodsell, D. S.; Olson, A. J. AutoDock4 and AutoDockTools4: Automated docking with selective receptor flexibility. J. Comput. Chem. 2009, 30, 2785-2791.

(92) Fry, D. W.; Harvey, P. J.; Keller, P. R.; Elliott, W. L.; Meade, M.; Trachet, E.; Albassam, M.; Zheng, X.; Leopold, W. R.; Pryer, N. K.; Toogood, P. L. Specific inhibition of cyclin-dependent kinase $4 / 6$ by
PD 0332991 and associated antitumor activity in human tumor xenografts. Mol Cancer Ther 2004, 3, 1427-1438.

(93) Gunther, S.; Kuhn, M.; Dunkel, M.; Campillos, M.; Senger, C.; Petsalaki, E.; Ahmed, J.; Urdiales, E. G.; Gewiess, A.; Jensen, L. J.; Schneider, R.; Skoblo, R.; Russell, R. B.; Bourne, P. E.; Bork, P.; Preissner, R. SuperTarget and Matador: Resources for exploring drugtarget relationships. Nucleic Acids Res. 2008, 36, D919-D922.

(94) Frey, B. J.; Dueck, D. Clustering by passing messages between data points. Science 2007, 315, 972-976.

(95) Bloom, J.; Pagano, M. Deregulated degradation of the cdk inhibitor p27 and malignant transformation. Semin. Cancer Biol. 2003, $13,41-47$.

(96) El Baroudi, M.; Cora, D.; Bosia, C.; Osella, M.; Caselle, M. A curated database of miRNA mediated feed-forward loops involving MYC as master regulator. PLoS One 2011, 6, e14742.

(97) Latora, V.; Marchiori, M. Efficient behavior of small-world networks. Phys. Rev. Lett. 2001, 87, 198701.

(98) Apweiler, R.; Bairoch, A.; Wu, C. H.; Barker, W. C.; Boeckmann, B.; Ferro, S.; Gasteiger, E.; Huang, H.; Lopez, R.; Magrane, M.; Martin, M. J.; Natale, D. A.; O’Donovan, C.; Redaschi, N.; Yeh, L. S. UniProt: The universal protein knowledgebase. Nucleic Acids Res. 2004, 32, D115-D119.

(99) Tuncbag, N.; Kar, G.; Gursoy, A.; Keskin, O.; Nussinov, R. Towards inferring time dimensionality in protein-protein interaction networks by integrating structures: the p53 example. Mol. Biosyst. 2009, 5, 1770-1778.

(100) Morris, J. H.; Apeltsin, L.; Newman, A. M.; Baumbach, J.; Wittkop, T.; Su, G.; Bader, G. D.; Ferrin, T. E. clusterMaker: A multialgorithm clustering plugin for Cytoscape. BMC Bioinf. 2011, 12, 436.

(101) Smoot, M. E.; Ono, K.; Ruscheinski, J.; Wang, P. L.; Ideker, T. Cytoscape 2.8: New features for data integration and network visualization. Bioinformatics 2011, 27, 431-432. 